\title{
Uncertainty Analysis for the Evaluation of a Passive Runway Arresting System
}

\author{
Richard DeLoach $^{*}$ and Jill M. Marlowe ${ }^{\dagger}$ \\ NASA Langley Research Center, Hampton, Virginia, 23681 \\ Thomas J. Yager ${ }^{\ddagger}$ \\ ATK Space Systems, Hampton, Virginia 23681
}

This paper considers the stopping distance of an aircraft involved in a runway overrun incident when the runway has been provided with an extension comprised of a material engineered to induce high levels of rolling friction and drag. A formula for stopping distance is derived that is shown to be the product of a known formula for the case of friction without drag, and a dimensionless constant between 0 and 1 that quantifies the further reduction in stopping distance when drag is introduced. This additional quantity, identified as the Drag Reduction Factor, D, is shown to depend on the ratio of drag force to friction force experienced by the aircraft as it enters the overrun area. The specific functional form of $D$ is shown to depend on how drag varies with speed. A detailed uncertainty analysis is presented which reveals how the uncertainty in estimates of stopping distance are influenced by experimental error in the force measurements that are acquired in a typical evaluation experiment conducted to assess candidate overrun materials.

\section{Nomenclature}

$=$ acceleration

$=$ Drag force

$=$ Initial drag force at entry into EMAS material

$=$ Friction force

$=$ acceleration of gravity

$=$ mass, and $\ln \left(F_{H} / f\right)$

$=$ Ratio of initial drag, $d_{0}$, to constant friction force, $f$

$=$ time

$=$ displacement

$A$

$=$ Reference area, related to the area of a projection of an object on the plane that is perpendicular to the direction of motion

$=$ Drag coefficient

$=$ Drag Reduction Factor

$=$ Horizontal force (friction plus drag)

$=$ Vertical force (load)

$=$ Proportionality constants

$=$ Stopping distance

$=$ Speed

$=$ Speed with which aircraft enters an EMAS runway extension

*Senior Research Scientist, Aeronautical Systems Engineering Branch, NASA Langley Research Center, MS 238, 4 Langley Blvd, Hampton, VA 23681, Associate Fellow.

${ }^{\dagger}$ Head, Structural Dynamics Branch, NASA Langley Research Center, MS 230, Hampton, VA 23681, Senior Member.

"Senior Research Engineer, ATK Space Systems, NASA Langley Facility, 1221B Langley Blvd, MS 186A, Hampton, VA 23681 
$\mu$

$\rho$

EMAS

FAA

LaRC

$M D O E$

$M T T F$

NASA

$P A N Y N J$

$P D F$

$S D B$

Standard error
$=$ Coefficient of friction

$=$ Density

$=$ Engineered Materials Arresting System

$=$ Federal Aviation Administration

$=$ Langley Research Center

$=$ Modern Design of Experiments

$=$ Mobile Tire Test Facility

$=$ National Aeronautics and Space Administration

$=$ Port Authority of New York and New Jersey

$=$ Probability Density Function

$=$ Structural Dynamics Branch

$=$ Square root of variance. The "one-sigma" estimate of dispersion

\section{Introduction}

$\mathrm{R}$ unway overruns present a serious threat to the safety of airline passengers and crew, and can also result in costly damage to aircraft. Examples of such overrun accidents include 1) Little Rock, AR, MD82 aircraft, June 1, 1999; 2) Toronto, Ontario, A340 aircraft, August 2, 2005; and 3) Chicago Midway, IL, B737 aircraft, December 8,2005 . Loss of runway friction was considered a contributing cause in each of these accidents.

Passive arresting systems have been studied as a countermeasure for loss of runway friction since the early $1960 \mathrm{~s}^{1-7}$. The most practical results have involved soft-ground materials of gradually increasing depth as the distance from the end of the paved runway threshold increases. Later studies ${ }^{8-11}$ considered foamed plastics, soils, foam cement, and quick-deploying net barriers.

In September 2005, the Federal Aviation Administration (FAA) issued Advisory Circular No. 150/5220-22A that discusses Engineered Materials Arresting Systems (EMAS) for aircraft overruns ${ }^{12}$. Such a system consists of a bed of energy absorbing material extending the normal operating range of an airport runway. The material passively decelerates an aircraft by inducing high levels of friction and drag as the aircraft landing gear gradually sinks into it. Displacement drag forces that are generated as the landing gear burrows through this material are applied through the distance the aircraft traverses, as are friction forces. The resulting work dissipates the kinetic energy of the aircraft until it eventually comes to rest.

Since the Midway Airport accident, more than 30 foam cement EMAS installations have been established as extensions of civil airport runways. Runway 4/22 at JFK International Airport received the first installation, soon after successful qualification tests were completed at the FAA Technical Center, Atlantic City, New Jersey in the 1990s. In the interim, successful arrestments of three different aircraft have occurred. The Port Authority of New York \& New Jersey (PANYNJ) has since been evaluating less costly candidate materials for an improved Engineered Materials Arresting System based on new, more durable materials that comply with the FAA Advisory Circular No. 150/5220-22A.

In late 2007, PANYNJ entered into an agreement with NASA Langley Research Center's Structural Dynamics Branch (SDB) to conduct field friction tests as part of their EMAS evaluation ${ }^{13}$. NASA has conducted research in the area of aircraft ground handling performance for several decades and has a strategic interest in improving aviation safety. Recently, SDB completed development of the Mobile Tire Test Facility (MTTF), shown in Fig. 1, which is designed to take friction measurements for tire/ground interactions at a variety of speeds and tire vertical loads and for a range of braking, steering, and rolling resistance test configurations.

The SDB approached Langley's Aircraft Systems Engineering Branch (ASEB) for certain testing technology support in conjunction with the proposed PANYNJ collaboration. Specifically, there was a request for a design of the test matrix to be used for field evaluations of candidate EMAS materials that could exploit the Modern Design of Experiments (MDOE), an integrated experiment design, execution, and analysis process that has been used for years by ASEB to maximize quality and productivity in empirical aerospace research at Langley and elsewhere ${ }^{14-19}$. An MDOE test matrix was executed using the MTTF on November 14, 2007 at a PANYNJ-designated site on the property of JFK International Airport. A comprehensive repor $\mathrm{t}^{20}$ describing results for the specific candidate EMAS materials under evaluation was prepared for delivery to the Port Authority in 2008.

The current paper focuses on a related issue that surfaced during the EMAS materials evaluation tests at JFK. Those results suggested that an initial hypothesis about the stopping mechanism was incorrect. We assumed initially that an aircraft wheel immersed in an EMAS runway extension would experience a net horizontal force consisting of the sum of friction and drag forces, and that the aircraft's kinetic energy in an overrun incident would be dissipated 
by the work done as this net horizontal force was applied through the stopping distance. We therefore believed that measurements of the total horizontal force would be sufficient to predict stopping distance.

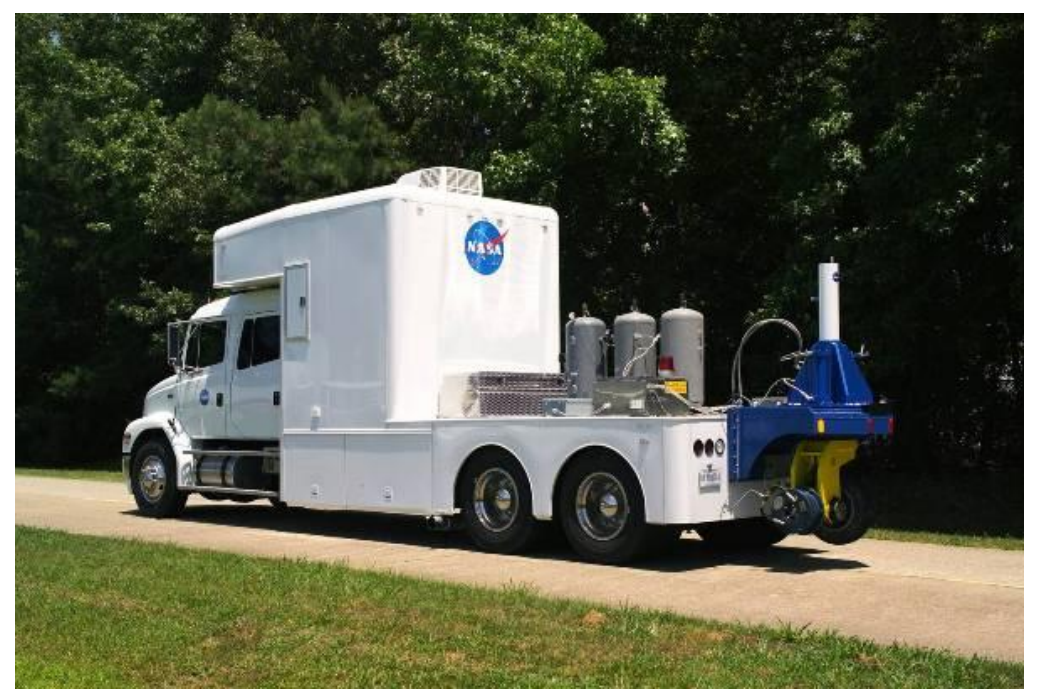

Figure 1. NASA's Mobile Tire Test Facility

Subsequent analysis suggests that while the work done to dissipate kinetic energy in a runway overrun incident is in fact the total horizontal force integrated over the stopping distance, the stopping distance itself is dependent on the ratio of drag force to friction force, rather than the simple sum of those two forces. This has important implications for how EMAS systems are evaluated because it suggests that friction and drag must be measured independently; a simple non-differentiated sum of the two forces is insufficient to assess stopping distance.

Furthermore, since drag forces are dependent on velocity while friction forces are not, the greater the initial velocity of an aircraft entering an EMAS runway extension the greater will be the difference in drag and friction effects. For a relatively high-speed EMAS evaluation run, drag measurement errors will therefore have a different impact on estimates of stopping distance than an equivalent experimental error in estimating the force of friction. The dependence of stopping distance on the ratio of drag to friction suggested that friction measurement errors might be especially important, even when drag dominates in a high-speed entry. The experimental error in both friction and drag force measurements could be estimated directly from the data, as could the experimental error in measurements of the applied vertical load. This paper describes how stopping distance depends on these loads, and how these different experimental errors propagate into the uncertainty in estimates of stopping distance.

The current paper begins with the derivation of a formula to predict stopping distance as a function of friction and drag forces for a specified EMAS entry speed. This is followed by an uncertainty analysis that reveals the relative impact of experimental errors in drag and friction on the uncertainty in stopping-distance forecasts.

The analysis is complicated by the fact that stopping distance depends on how the drag force varies with speed. An initial assumption of a simple Rayleigh quadratic speed dependence for drag was not supported by the data, which suggested a more nearly linear speed dependence that implied some variation of Stokes' drag. On the other hand, the EMAS evaluation runs were necessarily conducted over a relatively limited speed range due to practical real estate and cost constraints at JFK. These constraints foreclosed options for longer run-up and deceleration lanes, and longer EMAS test beds, that would have been necessary to accommodate testing at higher speeds. The dependence of drag on speed did appear to transition between first and second order as the rate of displacement of mass from the EMAS test beds increased, suggesting that the weaker speed dependence might have been an artifact of the low speeds dictated by resource constraints. Stopping distance formulae are therefore derived in this paper for both linear and quadratic speed dependencies. Both developments resulted in the same general functional form - $\mathrm{a}$ simple and well-known formula for friction-induced stopping distance that is multiplied by a dimensionless Drag Reduction Factor in the range of 0 to 1. The Drag Reduction Factor is a function of the ratio of drag force to friction force, and quantifies the further reduction in stopping distance that is achieved when frictional forces are augmented by drag.

For both speed dependencies, the uncertainty in stopping distance is estimated under an assumption of different experimental errors for high-force drag and low-force friction measurements. The uncertainty analysis reveals some 
unanticipated insights into the relative impact of friction and drag experimental errors on the precision of stopping distance estimates.

The paper is organized as follows: The introduction in this Section I is followed in Section II by a brief review of stopping distance in the absence of drag, when only the force of friction is in play. The uncertainty in estimating stopping distance under these conditions is also quantified. Section III extends the friction-only case to account for the effects of quadratic drag. The impact on stopping distance precision of experimental errors in the measurement of friction and drag is considered in Section IV. Section V reviews experimental results of the NASA/PANYNJ collaboration that reveal an unanticipated speed dependence for drag. The impact that the observed speed dependence would have on stopping distance is described in Section VI. Section VII contains a discussion of principal findings, which are summarized in Section VIII.

\section{Stopping Distance Due to Friction}

We will briefly postpone consideration of drag effects in order to illustrate the general approach to estimating stopping distance with a simpler case in which only friction is in play. We assume that an aircraft rolls into the EMAS material at some original velocity, $V_{0}$, and that this velocity decreases to zero over some range, $R$, the stopping distance. In the general case, retarding forces due to friction, $f$, and drag, $d$, act through the stopping distance, $R$, to perform work that dissipates the kinetic energy of the aircraft.

We assume level runways and neglect such potential contributors to the retarding force as air resistance, the effects of airborne or standing precipitation, and any active aircraft systems for reducing velocity such as brakes or reverse thrusters. We also neglect factors that may extend the stopping distance, such as hydroplaning over standing water and residual engine thrust. That is, we neglect all other factors besides friction and drag forces that are attributable to the EMAS material itself.

We compute the stopping distance, $R$, by integrating horizontal force over this distance to compute the corresponding work, and setting this equal to the kinetic energy of the aircraft. If the horizontal force is due only to friction, the force is independent of distance and is just the coefficient of friction, $\mu$, times the vertical force, which is simply the product of mass, $m$, times the acceleration of gravity, $g$. Thus, we have

$$
1 / 2 m V_{0}^{2}=\int_{x=0}^{x=R} F_{H} d x=m g \mu \int_{x=0}^{x=R} d x=m g \mu R
$$

We solve for $R$ and introduce the subscript $f$ to explicitly denote the stopping distance due only to rolling friction and not to displacement drag, obtaining this well-known result:

$$
R_{f}=\frac{V_{0}^{2}}{2 g \mu}
$$

For a given original velocity, $V_{0}$, the stopping distance due to rolling friction depends only on the coefficient of friction. Note that it does not depend on the mass of the aircraft. This is because the kinetic energy that must be dissipated, and the work generated by the force of friction to dissipate that energy, are each directly proportional to mass.

FAA Advisory Circular No. 150/5220-22A, referenced above, requires that an EMAS runway extension be capable of stopping an aircraft overrun with an initial speed of 70 knots, or about $81 \mathrm{mph}$. Specific friction coefficient values depend on the nature and condition of the runway surface and the tire, but tabulated values in the range of about 0.03 for hard rubber on asphalt are representative. From Eq. (2), the corresponding stopping distance over untreated asphalt for an aircraft with an initial speed of 70 knots would then be in excess of 7200 feet. Real estate constraints typically limit the available overrun range to a few hundred feet. For example, airports in the New York City area have as little as 250 to 400 feet available for some runway extensions. These figures highlight the need for an arresting system that will provide shorter stopping distances than would occur with an untreated extension of an asphalt runway. Concrete is often used for runways instead of asphalt. The stopping distance on concrete is longer than on asphalt because concrete does not deform as much. The friction coefficient is typically less by a factor of two to three, and the stopping distance, per Eq. (2), is correspondingly longer.

As part of the NASA collaboration with PANYNJ, the MTTF was used to acquire the data necessary to estimate the friction coefficient for a specimen of asphalt in a nearby parking lot. (Logistical considerations foreclosed 
options of testing on an asphalt runway.) The uncertainty in this estimate of friction coefficient was also quantified. This presented a common problem in uncertainty analysis, in which one wishes to know the uncertainty of some quantity that is a function of one or more other factors. The uncertainty in those factors is known, and one wishes to know how they propagate into uncertainty in the quantity of interest.

For a function of several variables, $y\left(x_{1}, x_{2}, \ldots, x_{n}\right)$, the following general error propagation formula is wellknown for the case in which all errors are independent ${ }^{21,22}$ :

$$
\sigma_{y}^{2}=\left(\frac{\partial y}{\partial x_{1}}\right)^{2} \sigma_{x_{1}}^{2}+\left(\frac{\partial y}{\partial x_{2}}\right)^{2} \sigma_{x_{2}}^{2}+\cdots+\left(\frac{\partial y}{\partial x_{n}}\right)^{2} \sigma_{x_{n}}^{2}
$$

In this case, $y$ is the stopping distance given in Eq. (2) and there is only one independent variable, $x_{1}=\mu$. Therefore, we have

$$
\frac{d R_{f}}{d \mu}=-\frac{V_{0}}{2 g \mu^{2}} \rightarrow \sigma_{R_{f}}^{2}=\left(\frac{-V_{0}}{2 g \mu^{2}}\right)^{2} \sigma_{\mu}^{2}
$$

or

$$
\sigma_{R_{f}}=R_{f}\left(\frac{\sigma_{\mu}}{\mu}\right)
$$

That is, the standard error in stopping distance due to random error in the friction coefficient estimate is just the nominal stopping distance times the standard random error in friction coefficient expressed as a fraction of reading. The simple inverse-proportional relationship between stopping distance and friction coefficient means that, expressed as a fraction of reading, the standard random error in stopping distance and the standard random error in friction coefficient are the same:

$$
\frac{\sigma_{R_{f}}}{R_{f}}=\frac{\sigma_{\mu}}{\mu}
$$

We estimated this ratio to be 0.019 for the asphalt data acquired at JFK, suggesting a standard error is the estimate of stopping distance over asphalt of less than $2 \%$ of reading.

\section{Impact of Quadratic Drag on Stopping Distance}

Displacement drag is the force that is associated with work performed when a wheel plows a furrow through the EMAS material. It is the work required to displace the EMAS material from the resulting rut. The energy required to perform this work is drawn from the kinetic energy of the aircraft, which reduces that energy and therefore reduces the speed of the aircraft. When displacement drag is in play as well as friction, the total horizontal force, $F_{H}$, is comprised of the displacement drag force, $d$, and the force of friction, $f$. In this section, we extend Eq. (2) to account for the impact of displacement drag on stopping distance.

\section{A. Stopping Distance with Drag and Friction}

The force of friction is well known to be the product of the vertical load times the coefficient of friction. We assume that the drag force is analogous to the aerodynamic drag experienced by an object in relatively high-speed motion through a fluid, known as Rayleigh Drag. Rayleigh Drag is also known as quadratic drag because of its dependence on the square of speed. We assume this particular form of displacement drag for the EMAS materials because of empirical results in runway friction tests that have shown a similar speed dependence for displacement drag due to water, slush, or snow accumulation ${ }^{23-25}$. 
We therefore have

$$
F_{H}=f+d
$$

where $F_{H}$ is the total horizontal force,

$$
f=-\mu F_{V}
$$

is the force of friction with $\mu$ as the friction coefficient and $F_{V}$ as the vertical force; and

$$
d=-1 / 2 \rho V^{2} A C_{D}
$$

is the displacement drag force, where $\rho$ is the density of the "fluid," $V$ is the speed, $A$ is a reference area that is related to the area of a projection of the object on the plane that is perpendicular to the direction of motion, and $C_{D}$ is the drag coefficient, a dimensionless constant analogous to the coefficient of friction. The minus signs in Eqs; (8) and (9) indicate that the friction and drag forces are opposite the opposite the direction of motion.

For a given vertical load, for which $A$ is a constant, we can rewrite displacement drag as

$$
d=-K V^{2}
$$

where for convenience we have rolled up a number of constants into $K$ as follows:

$$
K=1 / 2 \rho A C_{D}
$$

Combining Eqs. (7), (8), and (10) with Newton's second law yields this equation of motion for the vehicle traversing the EMAS material:

$$
F_{H}=m a=-K V^{2}-m g \mu
$$

The vehicle will stop when the force in Eq. (12) has been applied through a distance sufficient to generate an amount of work that consumes all of the available kinetic energy of the aircraft. That is, the condition for stopping the aircraft is

$$
1 / 2 m V^{2}=\int_{0}^{R} F_{H} d x
$$

This is the same formulation as the friction-only case, except for the added complication that drag is a function of velocity, which changes with distance. We must therefore express $V$ as a function of distance. We begin by expressing the equation for horizontal force as the following non-linear differential equation:

$$
m \frac{d^{2} x}{d t^{2}}=-K\left(\frac{d x}{d t}\right)^{2}-m g \mu
$$


or

$$
\frac{d^{2} x}{d t^{2}}+\left(\frac{K}{m}\right)\left(\frac{d x}{d t}\right)^{2}+g \mu=0
$$

But

$$
V=\frac{d x}{d t} \rightarrow \frac{d^{2} x}{d t^{2}}=\frac{d V}{d t}
$$

so

$$
\frac{d V}{d t}+\left(\frac{K}{m}\right) V^{2}+g \mu=0
$$

Apply the chain rule to the derivative representing acceleration:

$$
\frac{d V}{d t}=\left(\frac{d V}{d x}\right)\left(\frac{d x}{d t}\right)=V\left(\frac{d V}{d x}\right)
$$

Insert this into Eq. (17):

$$
V\left(\frac{d V}{d x}\right)+\left(\frac{K}{m}\right) V^{2}+g \mu=0
$$

or

$$
V d V=-\left[\left(\frac{K}{m}\right) V^{2}+g \mu\right] d x
$$

Divide by the bracketed term on the right:

$$
\frac{V d V}{\left[\left(\frac{K}{m}\right) V^{2}+g \mu\right]}=-d x
$$

We take the indefinite integral of both sides of this equation.

$$
\int \frac{V d V}{\left[\left(\frac{K}{m}\right) V^{2}+g \mu\right]}=-\int d x
$$


The integral on the right is, of course, simply $-x$. The integral on the left is of a general form that can be evaluated as follows ${ }^{26}$ :

$$
\int \frac{u d u}{\left(a u^{2}+b\right)}=\frac{1}{2 a} \ln \left(a u^{2}+b\right)
$$

In this case, $u=V, a=K / m$, and $b=g \mu$, so we have

$$
\left(\frac{m}{2 K}\right) \ln \left[\left(\frac{K}{m}\right) V^{2}+g \mu\right]=-x+\text { Const }
$$

where Const is a constant of integration. It is convenient to express Const as $\ln (C)$, which we can do with no loss of generality, where $C$ is simply some constant yet to be evaluated. The reason for expressing the constant of integration in this form will be clear shortly.

We thus have

$$
\left(\frac{m}{2 K}\right) \ln \left[\left(\frac{K}{m}\right) V^{2}+g \mu\right]=-x+\ln (C)
$$

Or

$\ln \left[\left(\frac{K}{m}\right) V^{2}+g \mu\right]=-\frac{2 K x}{m}+\frac{2 K}{m} \ln (C) \rightarrow \ln \left[\left(\frac{K}{m}\right) V^{2}+g \mu\right]-\frac{2 K}{m} \ln (C)=-\frac{2 K}{m} x$

We exploit two general properties of logarithms, as follows:

$$
a \ln (b)=\ln \left(b^{a}\right) \rightarrow-\frac{2 K}{m} \ln (C)=+\ln \left(C^{\frac{-2 K}{m}}\right)
$$

and

$$
\ln (a)+\ln (b)=\ln (a b) \rightarrow \ln \left[\left(\frac{K}{m}\right) V^{2}+g \mu\right]+\ln \left(C^{\frac{-2 K}{m}}\right)=\ln \left\{C^{\frac{-2 K}{m}}\left[\left(\frac{K}{m}\right) V^{2}+g \mu\right]\right\}
$$

Using these results in Eq. (26):

$$
\ln \left\{C^{\frac{-2 K}{m}}\left[\left(\frac{K}{m}\right) V^{2}+g \mu\right]\right\}=-\frac{2 K}{m} x \rightarrow C^{\frac{-2 K}{m}}\left[\left(\frac{K}{m}\right) V^{2}+g \mu\right]=e^{\frac{-2 K}{m} x}
$$

or

$$
\left(\frac{K}{m}\right) V^{2}+g \mu=C^{+\frac{2 K}{m}} e^{-\frac{2 K}{m} x}
$$


In this equation, $x$ measures the distance traveled by the aircraft since entering the EMAS material. At $x=0, V=V_{0}$, the original velocity of the aircraft upon entering the EMAS material. Therefore

$$
\frac{K V_{0}^{2}}{m}+g \mu=C^{\frac{2 K}{m}}
$$

Substituting this for $C^{2 K / m}$ in Eq. (29), we have:

$$
\left(\frac{K}{m}\right) V^{2}+g \mu=\left(\frac{K V_{0}^{2}}{m}+g \mu\right) e^{-\frac{2 K}{m} x}
$$

The expression on the left of Eq. (31) is just $F_{H} / m$, the horizontal force divided by the mass (i.e., the horizontal acceleration). Therefore:

$$
F_{H}=m\left(\frac{K V_{0}^{2}}{m}+g \mu\right) e^{-\frac{2 K}{m} x}=\left(K V_{0}^{2}+m g \mu\right) e^{-\frac{2 K}{m} x}
$$

The term on the right of Eq. (32) in parentheses is simply the sum of the drag and friction forces that the aircraft experiences the moment it enters the EMAS material. That is, it is the initial retarding force. Eq. (32) reveals that the horizontal force decays exponentially from this initial retarding force, getting smaller as the aircraft gets further into the material.

As in the prior case in which only friction was in play, we integrate the horizontal force, represented by Eq. (32), from 0 to $R$. We do this to compute the work done by this force over a distance $R$. When this work is sufficient to dissipate the original kinetic energy that the aircraft had as it entered the EMAS material, the aircraft will stop, and $R$ will be the stopping distance. Therefore, we have

$$
1 / 2 m V_{0}^{2}=\int_{x=0}^{x=R} F_{H} d x=\left(K V_{0}^{2}+m g \mu\right) \int_{x=0}^{x=R} e^{-\frac{2 K}{m} x} d x
$$

The integral on the right is of an elementary form that is easy to evaluate:

$$
\int e^{-\frac{2 K}{m} x} d x=-\frac{m}{2 K} e^{-\frac{2 K}{m} x} \rightarrow \int_{x=0}^{x=R} e^{-\frac{2 K}{m} x} d x=\frac{m}{2 K}\left(1-e^{-\frac{2 K}{m} R}\right)
$$

We therefore have the following as a condition of stopping:

$$
1 / 2 m V_{0}^{2}=\frac{m\left(K V_{0}^{2}+m g \mu\right)}{2 K}\left(1-e^{-\frac{2 K}{m} R}\right)
$$

which reduces to

$$
e^{-\left(\frac{2 K}{m}\right) R}=1-\frac{K V_{0}^{2}}{K V_{0}^{2}+m g \mu}=\frac{m g \mu}{K V_{0}^{2}+m g \mu}
$$


The term on the right of Eq. (36) is the fraction of the total retarding force attributable to friction. We take the natural logarithm of both sides to solve for $R$ :

$$
-\left(\frac{2 K}{m}\right) R=\ln \left(\frac{m g \mu}{K V_{0}^{2}+m g \mu}\right)
$$

We can express this as

$$
-\left(\frac{2 K}{m}\right) R=\ln \left[\frac{1}{1+\left(\frac{K V_{0}^{2}}{m g \mu}\right)}\right]=-\ln \left[1+\left(\frac{K V_{0}^{2}}{m g \mu}\right)\right]
$$

Solving Eq. (38) for R:

$$
R=\frac{m}{2 K} \ln \left[1+\left(\frac{K V_{0}^{2}}{m g \mu}\right)\right]
$$

\section{B. The Role of Friction}

The fact that drag forces tend to dominate friction forces, especially in the relatively more interesting cases of high EMAS entry speed, may suggest that friction forces are essentially negligible, and that it should be possible to estimate stopping distance to a very good approximation by ignoring friction forces altogether. However, Eq. (39) reveals that the stopping distance, $R$, is a function of the ratio of the drag force to the friction force. In the limit as the coefficient of friction approaches zero, the stopping distance approaches infinity, no matter how great the displacement drag is. The important role that friction plays in stopping an aircraft can be understood by recognizing that, absent friction in this model, the only force available to dissipate kinetic energy is one that depends on velocity. Recall Eq. (20), reproduced here for convenience:

$$
V d V=-\left[\left(\frac{K}{m}\right) V^{2}+g \mu\right] d x
$$

For $\mu=0$, this reduces to

$$
d V=-\left(\frac{K V}{m}\right) d x \rightarrow \frac{d V}{V}=-\left(\frac{K}{m}\right) d x
$$

Integrating both sides:

$$
\int \frac{d V}{V}=-\frac{K}{m} \int d x \rightarrow \ln (V)+\text { const }=-\left(\frac{K}{m}\right) x
$$

We express the integration constant as $\ln (\mathrm{C})$ with no loss of generality:

$$
\ln (V)+\ln (C)=\ln (C V)=-\left(\frac{K}{m}\right) x \rightarrow C V=e^{-\left(\frac{K}{m}\right) x}
$$


We evaluate Eq. (42) at $x=0$, where $V=V_{0}$ :

$$
C V_{0}=1 \rightarrow C=\frac{1}{V_{0}} \rightarrow \frac{V}{V_{0}}=e^{-\left(\frac{K}{m}\right) x}
$$

So, absent friction, we have from Eq. (43):

$$
V=V_{0} e^{-\left(\frac{K}{m}\right) x}
$$

That is, absent friction the velocity approaches zero exponentially with distance, but zero velocity remains an asymptotic limit, never reached. A finite coefficient of rolling friction ensures that the aircraft's velocity reaches zero in a finite distance. Recall Eq. (31):

$$
\left(\frac{K}{m}\right) V^{2}+g \mu=\left(\frac{K V_{0}^{2}}{m}+g \mu\right) e^{-\frac{2 K}{m} x}
$$

Solving for V:

$$
V=\sqrt{V_{0}^{2} e^{-\left(\frac{2 K}{m}\right) x}-\frac{m g \mu}{K}\left(1-e^{-\left(\frac{2 K}{m}\right) x}\right)}
$$

When $\mu=0$, Eq. (45) reduces to the decaying exponential velocity formula in Eq. (44) for the case of no friction, in which velocity only approaches zero in an asymptotic limit so that the stopping distance is infinite. But for $\mu \neq 0$, when $x=R, V=0$, and substituting these values and solving for $\mathrm{R}$ yields Eq. (39) for (a finite) stopping distance. This demonstrates that the only difference between a finite stopping distance and an infinite one is a non-zero coefficient of friction.

\section{Asymptotic Behavior of Stopping Distance Formula}

We require that the stopping distance formula (Eq. (39), reprinted here for convenience) give sensible results in certain limiting cases:

$$
R=\frac{m}{2 K} \ln \left[1+\left(\frac{K V_{0}^{2}}{m g \mu}\right)\right]
$$

Note, for example, that the stopping distance goes to zero as the original velocity, $V_{0}$, goes to zero. While not especially interesting per se, this is a necessary result for the stopping distance formula to be credible.

A more interesting case occurs when the ratio of drag force to friction force gets small but $V_{0}$ is not necessarily small. For this case $K=1 / 2 \rho A C_{D}$ has to be small, meaning that some or all of the other factors influencing drag besides velocity (EMAS material density, reference area, drag coefficient) are small. We would expect in this case that the general formula for stopping distance, Eq. (39), would approach the stopping distance formula in Eq. (2), which was derived for the case of no drag.

From the series representation for $\ln (1+a)$ :

$$
\ln (1+a)=a-\frac{a^{2}}{2}+\frac{a^{3}}{3}-\frac{a^{4}}{4}+\cdots=\sum_{i=1}^{\infty}(-1)^{i+1}\left(\frac{a^{i}}{i}\right)
$$


we have the following well-known approximation for small $a$ :

$$
\ln (1+a)=a
$$

Assuming small K, we can therefore rewrite Eq. (39) using Eq. (46) as follows:

$$
R_{\text {Small } K}=\frac{m}{2 K}\left(\frac{K V_{0}^{2}}{m g \mu}\right)=\frac{V_{0}^{2}}{2 g \mu}=R_{f_{0}}
$$

which is, indeed, Eq. (2), the formula for stopping distance when drag is negligible, for the case of $V=V_{0}$. We add the $f_{0}$ subscript to explicitly denote this as the stopping distance when only friction is in play, for the case in which $V$ is the original velocity upon entry into the EMAS material, $V_{0}$.

\section{The Drag Reduction Factor}

Equation (47), originally derived as Eq. (2), is a well-known formula for stopping distance when there is no displacement drag and only friction is in play. In this section we will show that generalizing the problem by introducing displacement drag simply extends this basic formula for stopping distance by a multiplicative factor that ranges between zero and one, representing the additional reduction in stopping distance attributable to drag. That is, we will show that the general formula for stopping distance can be cast in this form:

$$
R=\frac{V_{0}^{2}}{2 g \mu} D=R_{f_{0}} D
$$

where $D$, the Drag Reduction Factor, is a dimensionless function of the ratio of drag force to friction force that lies between zero and one.

We begin by noting that the general stopping distance formula, Eq. (39), can be expressed as follows:

$$
R=\frac{m}{2 K} \ln \left[1+\left(\frac{d_{0}}{f}\right)\right]
$$

where $d_{0}=K V_{0}^{2}$ is the initial displacement drag when the aircraft first enters the EMAS material, and $f=m g \mu$ is the constant friction force applied throughout the stopping distance. Note that the $m / 2 K$ factor in Eq. (49) can also be expressed in terms of these two forces:

$$
\frac{m}{2 K}=\left(\frac{m g \mu}{K V_{0}^{2}}\right)\left(\frac{V_{0}^{2}}{2 g \mu}\right)=\left(\frac{f}{d_{0}}\right) R_{f_{0}}
$$

Inserting Eq. (50) into Eq. (49):

$$
R=R_{f_{0}}\left\{\left(\frac{f}{d_{0}}\right) \ln \left[1+\left(\frac{d_{0}}{f}\right)\right]\right\}=\frac{V_{0}^{2}}{2 g \mu}\left\{\left(\frac{f}{d_{0}}\right) \ln \left[1+\left(\frac{d_{0}}{f}\right)\right]\right\}=\left(\frac{V_{0}^{2}}{2 g \mu}\right) D
$$


where we introduce $D$, the "Drag Reduction Factor":

$$
D=\left(\frac{f}{d_{0}}\right) \ln \left[1+\left(\frac{d_{0}}{f}\right)\right]
$$

The construction of the stopping distance formula in the form of Eq. (51) is particularly revealing. It shows that the effect of introducing displacement drag is to simply reduce the friction-only stopping distance by a factor of $D$. If we define $r$ to be the ratio of the initial displacement drag, $d_{0}$, to the constant friction force, $f$, we have

$$
D=\frac{\ln (1+r)}{r}
$$

The addition of drag must result in a non-negative stopping distance that is less than the friction-only case for any ratio of displacement drag to friction force. Therefore D must lie between zero and one for any value of $r$. To find out whether this is true or not, we evaluate $D$ in the limit as $r$ approaches zero and again in the limit as $r$ approaches infinity. The $D$ factor approaches an indeterminate form in both cases, but we can circumvent this by invoking L'Hopital's Rule, which states that as $x$ approaches some limit, $c$, the ratio of $f(x) / g(x)$ approaches the ratio of the derivatives of $f(x)$ and $g(x)$ if that ratio exists. Begin with the derivatives of the numerator and denominator of Eq. (53):

$$
\begin{aligned}
& \frac{d \ln (1+r)}{d r}=\frac{1}{1+r} \\
& \frac{d r}{d r}=1
\end{aligned}
$$

From the derivatives we have, by L'Hopital's Rule:

$$
\lim _{r \rightarrow c}\left[D=\frac{\ln (1+r)}{r}\right]=\lim _{r \rightarrow c}\left(\frac{1}{1+r}\right)=\left(\frac{1}{1+c}\right)
$$

We therefore have

$$
\lim _{r \rightarrow 0} D=1
$$

and

$$
\lim _{r \rightarrow \infty} D=0
$$

so $0 \leq D \leq 1$ for all values of $r=d_{0} f f$, which, by Eq. (48), means that the addition of any non-zero displacement drag will always reduce stopping distance from the friction-only case, as required.

\section{Impact of Experimental Force Measurement Errors}

The stopping distance, $R$, has been shown to be the product of two quantities, the friction-only stopping distance for the EMAS entry speed, $V_{0}$, and the Drag Reduction Factor. Each is a function of the ratio of two forces. The friction-only stopping distance for a given initial speed depends only on the coefficient of friction, $\mu$, which is the 
ratio of the force of friction, $f$, to the vertical load, $F_{V}$. The drag reduction factor depends only on the ratio of the drag force to the force of friction.

It is only possible to directly measure the force of friction on a test wheel with a given vertical load by measuring the horizontal force it experiences as it traverses a candidate EMAS material under conditions of negligible drag. A detailed discussion of the techniques for making such a friction-only measurement are beyond the scope of this paper, but one general method might be to execute one or more preliminary high-speed runs intended to evacuate a rut in the EMAS material, followed by a run at a specified vertical load in the same test bed, but at micro-speeds. Since drag is a function of speed and also of the frontal area of EMAS material that the wheel encounters, the combination of very low speed and a pre-evacuated rut should result in drag forces that are low enough to be neglected if the speed is sufficiently low. The horizontal force measured under these conditions can then be attributed to friction. The ratio of this friction force to the vertical load applied when it is measured represents an empirical estimate of the coefficient of friction, $\mu$.

Drag forces cannot be measured directly, since any practical conditions that induce drag will also induce friction. Only the total horizontal force, $F_{H}$, consisting of the sum of friction and drag, can be measured directly. However, if preliminary low-force micro-speed measurements of the horizontal force in an evacuated EMAS rut have resulted in reliable estimates of the friction coefficient, then the force of friction can be estimated even for high-speed runs dominated by drag. One need only multiply the friction coefficient by the vertical load applied during those runs. These values of the friction force can then be subtracted from total horizontal force measurements made at high speed to yield an experimental estimate of the drag force. The friction coefficient is independent of speed under conditions of interest (no braking, etc), so the drag force can be estimated for any speed by this method. With an adequate estimate of both drag and friction, the quantity $r$ can then be estimated, and thus the Drag Reduction Factor.

Because the friction-only stopping distance, $R_{f_{0}}$, and the Drag Reduction Factor, D, both depend on forces that are measured in an EMAS evaluation experiment, the precision of the stopping distance estimated via Eq. (48) will depend on experimental force measurement errors. This section quantifies how such measurement errors propagate into the uncertainty in stopping distance, and which experimental errors have the greatest impact.

The friction coefficient, $\mu$, is defined as $\mu=f / F_{V}$, so the friction-only stopping distance at $V=V_{0}$ can be expressed in terms of forces that are directly measured as follows:

$$
R_{f_{0}}=\frac{V_{0}^{2} F_{V}}{2 g f}
$$

The relatively low-level friction force measurements, whether made with the technique outlined above or some other method, can utilize instrumentation scaled to smaller ranges than required for large-force measurements of vertical load and high-speed horizontal forces when drag is in play. This would be recommended to maximize the quality of the low-force measurements, in which case the friction force measurements would be expected to have a different experimental error than the high-force vertical and horizontal force measurements. This distinction is taken into account in the subsequent analysis.

Since $d=F_{H}-f$, the drag/friction ratio, $r=d / f$, can also be expressed in terms of forces that are directly measured:

$$
r=\frac{d}{f}=\frac{F_{H}-f}{f}
$$

and we also have

$$
1+r=1+\frac{F_{H}-f}{f}=\frac{f+F_{H}-f}{f}=\frac{F_{H}}{f}
$$


The Drag Reduction Factor, $D$, can then also be expressed as a function of measured forces. Assume that we are only interested in the uncertainty in stopping distance under operational conditions in which drag dominates and frictional forces make a negligible contribution to the total horizontal force. In that case, $F_{H}>>f$; i.e., $r>>1$, and:

$$
r \approx 1+r \approx \frac{F_{H}}{f}
$$

Then

$$
D=\frac{\ln (1+r)}{r} \approx \frac{\ln (r)}{r}=\frac{f}{F_{H}} \ln \left(\frac{F_{H}}{f}\right)
$$

and

$$
R=R_{f_{0}} D=\frac{V_{0}^{2} F_{V}}{2 g f}\left(\frac{f}{F_{H}}\right) \ln \left(\frac{F_{H}}{f}\right)=\lambda\left(\frac{F_{V}}{F_{H}}\right) \ln \left(\frac{F_{H}}{f}\right)
$$

where we have introduced the symbol $\lambda=\frac{V_{0}^{2}}{2 g}$ to simplify the notation. Note that Eq. (61) is simply a generalized extension of the friction-only stopping distance formula of Eq. (56) to include drag, with $F_{H}=d+f$ replacing $f$ in the stopping distance formula. For $d=0$, Eq. (61) reverts to Eq. (56). One seemingly valid approach to estimating stopping distance in the presence of drag is to use the same technique as when drag is not in play, that is, to divide the quantity $\lambda$ by a friction coefficient estimated from the total measured horizontal force (drag plus friction) rather than friction alone. However, Eq (61) illustrates that if the horizontal force includes drag as well as friction, the resulting calculation must be multiplied by a correction factor consisting of the log of the total horizontal force expressed as a multiple of friction, which is just the log of 1 plus the drag/friction ratio.

From Eq. (61) we see that the estimate of stopping distance for a specified EMAS entry speed, $V_{0}$, depends on three measured forces; the vertical load, $F_{V}$, and values of horizontal force under two conditions, when drag is negligible $(f)$ and when it is not $\left(F_{H}\right)$. The impact that experimental errors in these measurements have on the uncertainty in the stopping distance estimate can be calculated as before, using the standard error propagation given in Eq. (3), which for this case can be expressed as

$$
\sigma_{R}^{2}=\left(\frac{\partial R}{\partial F_{V}}\right)^{2} \sigma_{F_{V}}^{2}+\left(\frac{\partial R}{\partial F_{H}}\right)^{2} \sigma_{F_{H}}^{2}+\left(\frac{\partial R}{\partial f}\right)^{2} \sigma_{f}^{2}
$$

To facilitate the differentiation, we rewrite Eq. (61) as follows:

$$
R=\lambda\left(\frac{F_{V}}{F_{H}}\right) \ln \left(\frac{F_{H}}{f}\right)=\lambda F_{V} F_{H}^{-1}\left[\ln \left(F_{H}\right)-\ln (f)\right]
$$

The derivatives of $R$ with respect to each of the measured forces are now computed, to be inserted into Eq. (62).

$$
\frac{\partial R}{\partial F_{V}}=\left(\frac{\lambda}{F_{H}}\right) \ln \left(\frac{F_{H}}{f}\right)
$$




$$
\begin{aligned}
\frac{\partial R}{\partial F_{H}} & =\lambda F_{V}\left\{\left[\ln \left(F_{H}\right)-\ln (f)\right]\left(-F_{H}^{-2}\right)+F_{H}^{-1}\left(\frac{1}{F_{H}}\right)\right\} \\
& =\lambda F_{V}\left[\left(\frac{-1}{F_{H}^{2}}\right) \ln \left(\frac{F_{H}}{f}\right)+\frac{1}{F_{H}^{2}}\right]=\frac{\lambda F_{V}}{F_{H}^{2}}\left[1-\ln \left(\frac{F_{H}}{f}\right)\right] \\
\frac{\partial R}{\partial F_{H}} & =\left(\frac{\lambda}{F_{H}}\right)\left\{\left(\frac{F_{V}}{F_{H}}\right)\left[1-\ln \left(\frac{F_{H}}{f}\right)\right]\right\} \\
\frac{\partial R}{\partial f} & =-\left(\frac{\lambda}{F_{H}}\right)\left(\frac{F_{V}}{f}\right)
\end{aligned}
$$

As noted above, estimating stopping distance from force measurements in an EMAS material evaluation experiment entails essentially two classes of force measurements, which we may describe as "large forces" and "small forces." Large forces include the total horizontal force at high-speed entry into the EMAS material and the vertical loads that simulate the aircraft weight per wheel. These are expected to be on the order of thousands of pounds in a typical evaluation experiment involving the FAA-mandated evaluation speed of 70 knots. By contrast, the force of friction under conditions of negligible drag, $f$, will be proportional to the vertical load, with the coefficient of friction, $\mu$, as a dimensionless proportionality constant. Specific values of $\mu$ will depend on the EMAS material but values on the order of several hundredths would be anticipated, meaning that frictional forces would be one or two orders of magnitude smaller than the vertical load forces - perhaps a few hundred pounds at most. As noted above, the most accurate measurements of these smaller forces would require force instrumentation scaled to much smaller ranges than the instruments that would be used to measure the large vertical loads and high-speed drag forces. We assume for this analysis, then, that the experimental force measurement errors can be characterized as those that accompany the large force measurements $\left(F_{H}\right.$ and $\left.F_{V}\right)$, and those that accompany the small force measurements $(f)$.

We introduce the following nomenclature for the large-force experimental errors, under the assumption that the uncertainty in horizontal and vertical large-force measurements is the same:

$$
\sigma_{F}^{2}=\sigma_{F_{V}}^{2}=\sigma_{F_{H}}^{2}
$$

We also introduce this simplifying nomenclature:

$$
m=\ln \left(\frac{F_{H}}{f}\right)
$$

Insert Eqs. (64) - (68) into Eq. (62):

$$
\begin{gathered}
\sigma_{R}^{2}=\left(\frac{\lambda}{F_{H}}\right)^{2}\left\{\left[m^{2}+(m-1)^{2}\left(\frac{F_{V}}{F_{H}}\right)^{2}\right] \sigma_{F}^{2}+\left(\frac{F_{V}}{f}\right)^{2} \sigma_{f}^{2}\right\} \\
\sigma_{R}^{2}=\left(\frac{\lambda}{F_{H}}\right)^{2}\left\{\left[m^{2}+(m-1)^{2}\left(\frac{F_{V}}{F_{H}}\right)^{2}\right] \sigma_{F}^{2}+\left(\frac{1}{\mu}\right)^{2} \sigma_{f}^{2}\right\}
\end{gathered}
$$


The variance in stopping distance is seen to be the weighted sum of the variances in large- and small-force measurements, with the small-force weighting much larger than the large-force weighting. Therefore a given experimental error in the small-force measurements will contribute significantly more uncertainty to the stopping distance estimate than a similar error in the large-force measurements.

To see this, consider first the large-force weighting, represented by the term in square brackets. Vertical and horizontal forces are expected to be of comparable orders of magnitude so their ratio will be of order one. The quantity $m$ is the logarithm of a force ratio. Because the logarithm is such a weak function of its argument, $m$ will also be small for even the largest force ratios anticipated under practical conditions. So the magnitude of the entire weighting factor for large-force variance is expected to be relatively small-on the order of 10 for typical force levels.

By contrast, the weighting for the small-force variance is the square of a large ratio of forces. Specifically, it is the square of the ratio of the relatively large vertical load to the relatively small friction force, where that ratio is just the reciprocal of the coefficient of friction. The coefficient of friction will vary from one EMAS material to another, but can be expected to be on the order of $10^{-1}$ or less. The square of its reciprocal will therefore be on the order of $10^{2}$ or greater, which is at least an order of magnitude greater than a typical weighting for the large-force variance.

We therefore reach a somewhat paradoxical conclusion, which is that notwithstanding how small the force of friction is compared to the drag induced by an EMAS material, a precise estimate of stopping distance is much more dependent upon high-precision friction measurements than on the quality of the drag measurements. In fact, the smaller the magnitude of the friction force relative to the vertical load, the more the uncertainty in stopping distance depends on the experimental error in estimating the friction force.

Because of the relative weightings, we can achieve a very good approximation to the uncertainty in stopping distance by simply ignoring the contribution due to large-force experimental error. Eq. (68) then reduces to

$$
\sigma_{R}^{2}=\left[\left(\frac{\lambda}{F_{H}}\right)\left(\frac{F_{V}}{f}\right)\right]^{2} \sigma_{f}^{2}
$$

The coefficient of friction is just the drag-free horizontal force, $f$, divided by the vertical load, $F_{V}$, so Eq. (70) can be rewritten as follows:

$$
\sigma_{R}^{2}=\left(\frac{\lambda}{\mu F_{H}}\right)^{2} \sigma_{f}^{2}
$$

But $\lambda / \mu$ is just $R_{f_{0}}$, the stopping distance when only friction is in play. So Eq. (71) reduces to:

$$
\sigma_{R}=\left(\frac{R_{f_{0}}}{F_{H}}\right) \sigma_{f}
$$

Thus, the standard error in stopping distance is directly proportional to the standard error in friction force and for practical purposes is essentially independent of the uncertainty in drag, notwithstanding how much greater a role drag plays in the total horizontal retarding force of an EMAS material than friction. This is attributable to the dependence of stopping distance on the ratio of drag to friction, with small errors in estimates of the relatively small denominator having substantial influence on the value of the force ratio and therefore the stopping distance.

\section{Empirical observations of the Dependence of Drag on Velocity}

During the NASA/PANYNJ collaboration, the MTTF shown in Fig. 1 was used to make horizontal force measurements on an aircraft test tire at two speeds and at two loads for each of two candidate EMAS materials that differed chiefly in their density. Low speed, low-load runs with a very shallow immersion depth generated horizontal forces attributed to friction, with drag assumed to be negligible. The coefficient of friction was computed for each candidate EMAS material by dividing these low-speed, low-immersion horizontal force measurements by the applied load on the test tire, which could be varied by a pneumatic pressure system built into the MTTF for this 
purpose. The speed-independent friction forces estimated in this way were subtracted from total horizontal force measurements made at higher speeds, where drag was the dominant mechanism. The horizontal force net of friction forces was attributed to drag.

The dependence of drag on speed was estimated by dividing drag measurements made under identical conditions except for speed. A linear speed dependence would have resulted in a drag ratio equal to the ratio of speeds. A quadratic speed dependence would have resulted in a drag ratio equal to the square of that ratio.

Speeds were measured using a ground-speed radar system, with measurements acquired at a rate of 50 per second. Mean and standard deviations of speed were estimated from these raw data.

The error propagation formula in Eq. (3) can be used to develop a formula for the standard error in a ratio of two quantities for which the standard error in each is known:

$$
y=\frac{x_{1}}{x_{2}} \rightarrow \sigma_{y}=\frac{\sqrt{\sigma_{x_{1}}^{2}+y^{2} \sigma_{x_{2}}^{2}}}{x_{2}}
$$

Using Eq. (73) and the means and standard deviations for low-speed and high-speed runs enabled us to estimate speed ratios as well as the standard error in the estimate of such ratios.

We can also apply Eq. (73) to estimate the ratio of two drag measurements and the standard error in estimating that ratio if we know the standard error in each of them. To estimate the standard error in drag, $d$, recall that drag is determined by subtracting an estimate of the force of friction, $f$, from measured horizontal force measurements, $F_{H}$. Again, Eq. (3) is used to estimate the standard error in such a differential measurement:

$$
d=F_{H}-f \rightarrow \sigma_{d}=\sqrt{\sigma_{F_{H}}^{2}+\sigma_{f}^{2}}
$$

The force of friction is estimated from measured values of the vertical load, $F_{V}$, and the coefficient of friction, $\mu$, as follows:

$$
f=\mu F_{V}
$$

and we again invoke Eq. (3), applying it to Eq. (75) to estimate the standard error in the estimate of friction force:

$$
\sigma_{f}=\sqrt{\left(\frac{\partial f}{\partial \mu}\right)^{2} \sigma_{\mu}^{2}+\left(\frac{\partial f}{\partial F_{V}}\right)^{2} \sigma_{F_{V}}^{2}}=\sqrt{F_{V}^{2} \sigma_{\mu}^{2}+\mu^{2} \sigma_{F_{V}}^{2}}
$$

Combine Eqs. (76) and (74) to express the standard error in drag as a function of the standard errors in horizontal and vertical force measurements and the standard error in the estimate of friction coefficient:

$$
\sigma_{d}=\sqrt{\sigma_{F_{H}}^{2}+F_{V}^{2} \sigma_{\mu}^{2}+\mu^{2} \sigma_{F_{V}}^{2}}
$$

As with the speed measurements, horizontal and vertical force measurements were made at the rate of 50 per second, permitting a direct estimate of both mean values and standard errors for each run. The standard error for friction coefficient, $\sigma_{\mu}$, was computed by applying Eq. (73) to the low-speed, shallow-immersion runs for which drag could be neglected. In those cases, $x_{1}$ and $x_{2}$ represented horizontal force, $F_{H}$, and load or vertical force, $F_{V}$, respectively. Thus, the standard error in each drag measurement could be computed using Eq. (77), and then Eq. (73) could be used to estimate the standard error in the ratio of two drag measurements. 
To test whether the dependence of drag on speed is linear or quadratic by comparing ratios of drag measurements to ratios of speed and the square of speed, we need to know the standard error in the square of the ratio of two quantities for which the standard error in each is known. Again invoke Eq. (3) to generate the following result:

$$
y=\left(\frac{x_{1}}{x_{2}}\right)^{2} \rightarrow \sigma_{y}=\frac{2 y}{x_{2}} \sqrt{\frac{\sigma_{x_{1}}^{2}}{y}+\sigma_{x_{2}}^{2}}
$$

We are now in a position to test whether the ratio of two drag measurements is the same as the ratio of their corresponding speeds, or if the drag ratio is the same as the ratio of their corresponding speeds squared. The former result implies a linear dependence of drag on speed; the latter implies a quadratic speed dependence.

We applied these tests to low- and high-speed runs through two candidate EMAS materials. Under the terms of the Space Act Agreement between NASA and the PANYNJ, neither the data nor specific descriptions of the candidate EMAS materials can be published until two years after the Agreement went into effect, in October of 2007. That will be approximately 10 months after the publication date of the current paper. Force and speed data are not included in this report for that reason, nor are descriptions of the specific EMAS materials that were tested, except to say that one was of relatively low density while the other was of a somewhat higher density. Within the constraints on early publication of proprietary data, it can be said that the ratio of measured mean speeds for the lowdensity EMAS material runs was $4.4 \pm 0.6$, per Eq. (73). The ratio of the square of mean speeds, per Eq. (78), was $19.0 \pm 2.2$. The ratio of drag forces corresponding to the low- and high-speed runs for the lower density EMAS material was $5.8 \pm 1.4$, again applying Eq. (73) to the drag measurements. These results are summarized in Table 1.

Table 1. Drag and speed ratios for lower-density EMAS material

\begin{tabular}{|l|c|c|}
\hline \multicolumn{1}{|c|}{ Quantity } & Value & Standard Error ("one sigma") \\
\hline Ratio of Speeds & 4.4 & 0.6 \\
\hline Squared Ratio of Speeds & 19 & 2.2 \\
\hline Ratio of Drag Measurements & 5.8 & 1.4 \\
\hline
\end{tabular}

We can formally test the two null hypotheses of interest:

$\mathrm{H}_{01}: d_{1} / d_{2}-V_{1} / V_{2}=0$, implying a linear speed dependence

$\mathrm{H}_{02}: \mathrm{d}_{1} / d_{2}-\left(V_{l} / V_{2}\right)^{2}=0$, implying a quadratic speed dependence

To test the first hypothesis for the lower-density EMAS material, invoke Eq. (3) for the general case of the difference in two quantities for which the standard error in each is known:

$$
y=y_{2}-y_{1} \rightarrow \sigma_{y}=\sqrt{\sigma_{y_{1}}^{2}+\sigma_{y_{2}}^{2}}
$$

Using Eq. (79) and the data from Table 1, we can test the first null hypothesis as follows:

$$
y=\frac{d_{1}}{d_{2}}-\frac{V_{1}}{V_{2}}=5.8-4.4=1.4, \quad \sigma_{y}=\sqrt{1.4^{2}+0.6^{2}}=1.5
$$

The difference in drag and speed ratios is not large compared to the standard error in estimating the difference, so we cannot reject the null hypothesis that the drag ratio is the same as the speed ratio and conclude that the drag exhibits a linear speed dependence in the lower-density EMAS material. 
The second hypothesis is tested in the same way and gives a consistent result:

$$
y=\frac{d_{1}}{d_{2}}-\left(\frac{V_{1}}{V_{2}}\right)^{2}=5.8-19.0=-13.2, \quad \sigma_{y}=\sqrt{1.4^{2}+2.2^{2}}=2.6
$$

The magnitude of the difference between the ratio of drag measurements and the ratio of the square of the corresponding speed measurements is large compared to the standard error in estimating that difference, so we reject the null hypothesis that the drag ratio is the same as the square of the speed ratio and conclude that the drag does not exhibit a quadratic speed dependence in the lower-density EMAS material. This analysis is performed graphically in Fig 2.

Figure displays probability density functions (PDFs) featuring means and standard deviations from Table 1 . The PDF on the left shows the distribution of speed ratios for low- and high-speed runs in the lower-density EMAS material. This is the distribution of drag ratios one would expect if drag varied linearly with speed. The PDF on the right in Fig. 2 represents the distribution of drag ratios that one would expect if there was a quadratic dependence of drag on speed in the lower-density EMAS material. It is broader than the PDF for the linear speed ratio because there is more uncertainty in the estimate of the square of the ratio of two random variables than in the linear ratio of the same variables, and it is shorter because the area of all PDFs sum to 1. The PDF in the middle represents the actual observed drag ratio for the lower-density EMAS material.

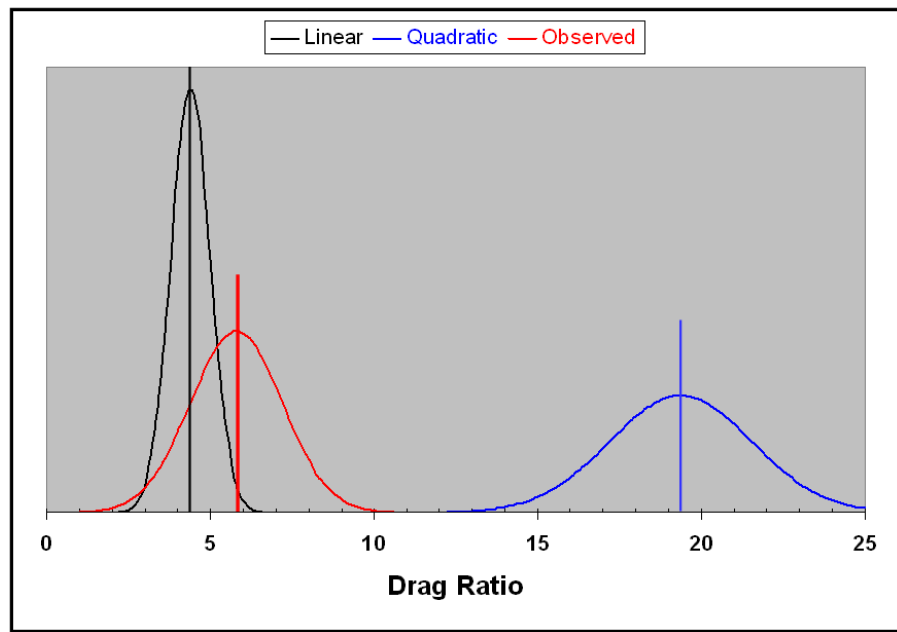

Figure 2. Drag ratio (red), ratio of speeds (black), and ratio of squared speeds (blue) for the lower-density EMAS material

Figure shows that the observed ratio of high-speed drag to low-speed drag in the low-density EMAS material can be unambiguously resolved from the square of the ratio of observed speeds. From this we infer that quadratic drag is not in play as we assumed above when we derived the formula for stopping distance. On the other hand, the PDF corresponding to the observed drag ratio overlaps the PDF for the linear ratio of speeds to such a degree that we cannot resolve the two distributions with high confidence. We are therefore unable to reject the hypothesis that in

the low-density EMAS material, displacement drag is a linear function of speed, but we are able to reject the hypothesis that displacement drag is a quadratic function of speed.

The weak speed dependence for drag observed in the lower-density EMAS material suggests a Stokes' Drag phenomenon. Stokes' Drag is experienced by relatively small objects moving at relatively low speeds. It is directly proportional to speed, with a proportionality constant that depends on the dimensions of the object and certain properties of the medium in which it moves, such as its viscosity. 
Because the empirical evidence of a linear speed dependence of drag upon speed in the lower-density EMAS material calls into question the stopping distance formula developed previously and given in Eq. (48), we performed a similar analysis for the higher-density EMAS material. The same two hypotheses were tested as for the lowerdensity EMAS material, this time using the data for the higher-density EMAS material that is summarized in Table 2.

Table 2. Drag and speed ratios for higher-density EMAS material

\begin{tabular}{|l|c|c|}
\hline \multicolumn{1}{|c|}{ Quantity } & Value & Standard Error (“one sigma") \\
\hline Ratio of Speeds & 4.0 & 0.5 \\
\hline Squared Ratio of Speeds & 16.0 & 2.1 \\
\hline Ratio of Drag Measurements & 7.4 & 1.0 \\
\hline
\end{tabular}

We again use Eq. (79) to test the first null hypothesis, as follows:

$$
y=\frac{d_{1}}{d_{2}}-\frac{V_{1}}{V_{2}}=7.4-4.0=3.4, \quad \sigma_{y}=\sqrt{1.0^{2}+0.5^{2}}=1.1
$$

Unlike the case of the lower-density EMAS material, the difference in drag and speed ratios is in fact larger than the standard error in estimating it, so we reject the null hypothesis that the drag ratio is the same as the speed ratio and tentatively conclude that the drag does not exhibit a linear speed dependence in the higher-density EMAS material. Because the drag ratio is greater than the speed ratio, the speed dependence appears to be stronger than linear, although this conclusion may be based on a weakness in the experimental method used to estimate drag, as will be discussed below.

The second hypothesis is tested in the same way:

$$
y=\frac{d_{1}}{d_{2}}-\left(\frac{V_{1}}{V_{2}}\right)^{2}=7.4-16.0=-8.6, \quad \sigma_{y}=\sqrt{1.0^{2}+2.1^{2}}=2.3
$$

The magnitude of the difference between the ratio of drag measurements and the ratio of the square of the corresponding speed measurements is large compared to the standard error in estimating that difference, so we reject the null hypothesis that the drag ratio is the same as the square of the speed ratio and again conclude that the drag does not exhibit a quadratic speed dependence in the lower-density EMAS material.

Figure compared linear and quadratic speed ratios with the observed drag ratio in the lower-density EMAS material, using the data summarized in Table 1. Figure 3. Drag ratio (red), ratio of speeds (black), and ratio of squared speeds (blue) for the higher-density EMAS material does the same for the higher-density material, using the data in Table 2. As in Fig. 2, the PDF on the left in Fig. 3 shows the distribution of speed ratios for low- and high-speed runs. Again, this is the distribution of drag ratios one would expect if drag varied linearly with speed. The PDF on the right in Fig. 3 represents the distribution of drag ratios for a quadratic dependence of drag on speed. The PDF in the middle represents the actual observed drag ratio for the higher-density EMAS material.

Figure shows that the observed ratio of high-speed drag to low-speed drag in the higher-density EMAS material can be unambiguously resolved from the ratio of the square of the speed ratio, just as was the case for the lowerdensity EMAS material. However, the higher-density results in Fig. 3 differ from the lower density results in Fig. 2 in that the drag ratio appears to also differ from the linear ratio of speeds. The first impulse is to infer from this that for the higher-density EMAS material, drag is neither a quadratic function of speed nor a linear function of speed, but something in between. However, this conclusion may be the result of a weakness in our technique for estimating drag, as will now be outlined.

The technique used to estimate friction was to rely on runs at low applied load that resulted in relatively shallow immersion depths. However, the immersion depth was never zero so drag forces were not eliminated entirely, as there was always some frontal area of EMAS material presented to the test wheel. This means that friction force estimates were probably overstated, and drag estimates made by subtracting these somewhat inflated friction values from a given horizontal force were therefore probably understated. 


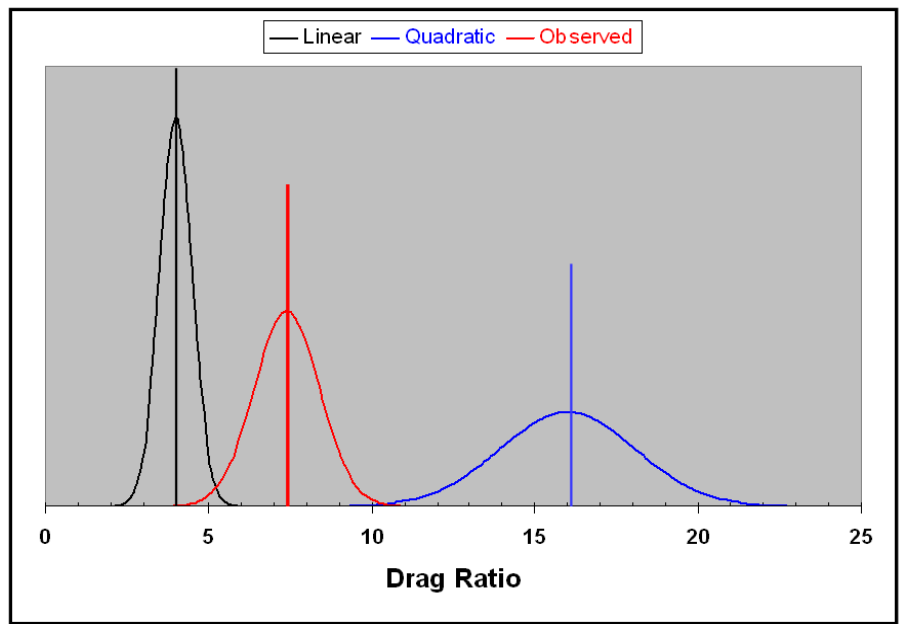

Figure 3. Drag ratio (red), ratio of speeds (black), and ratio of squared speeds (blue) for the higher-density EMAS material

Friction forces were estimated for the low-density EMAS material only after confirming that there was no significant difference between low-speed and high-speed horizontal forces measured at a very shallow immersion depths. Since drag depends on speed and friction does not, this implied negligible drag and suggested that the horizontal forcers are due only to friction.

Low load (i.e., shallow immersion) friction forces were only estimated for the high-density EMAS material at low-speed. The high-speed run was an outlier, generated from the repeat of a failed run executed earlier in the same test trench. Therefore there was no independent evidence that horizontal forces were the same for low and high speeds and therefore that drag was negligible during the high-density friction estimate. The high-density friction estimate might therefore have been more overstated than the low-density friction estimate. The increased density would argue for this as well, since the greater mass of material presented to a test wheel with the same immersion depth would have generated some additional drag.

At high load (non-negligible immersion depth so finite drag), any overstatement of friction would understate the drag estimates by a greater percentage at low speed (low drag) than at high speed (high drag). So the effect of overstating friction would be to produce a stronger apparent speed dependence than truly exists. This may explain why the drag ratio for the higher-density EMAS material, while still significantly less than the ratio of squared speeds, was nonetheless somewhat greater than the linear speed ratio. It is possible that the speed dependence was actually linear for the higher-density material just as it appears to have been for the lower-density material, but that there is an apparent elevated speed dependence that is attributable to overstated friction estimates, as described.

While Fig. 2 implies that the speed dependence for drag cannot be unambiguously distinguished from linear in the low-density material, the estimated speed dependence in fact does appear to be slightly greater than first-order (The mean of the red PDF in Fig. 2 is to the right of the mean of the black PDF, not withstanding considerable overlap of the two distributions). Again, this may be evidence of somewhat inflated friction force estimates due to non-negligible immersion depths even at low load.

The fact that the higher-density data seems to forecast a stronger speed dependence than the lower-density data is consistent with the notion that apparent departures from a first-order speed dependence may be due to the experimental technique described above. This would constitute further evidence of linear Stokes drag rather than quadratic Rayleigh drag. In any case, there does not seem to be any empirical evidence to support a quadratic speed dependence for drag.

To summarize the analysis of displacement drag's dependence on speed in the two EMAS materials, it appears to be a linear phenomenon (similar to Stokes' Drag) in the lower-density material, and may or may not have a stronger-than-linear speed dependence in the more dense material. In any case, there is no evidence to suggest that displacement drag is a quadratic function of speed for the loads, immersion depths, speed range, and materials of the PANYNJ EMAS evaluation experiment. We therefore re-derive the stopping distance formula in the next section assuming a linear speed dependence for drag instead of the quadratic speed dependence that was assumed when the 
stopping distance formula was initially derived. This alternative derivation reveals a somewhat surprising result, which is that the form of the stopping distance formula is quite similar for quadratic and linear speed dependencies. Only the form of the Drag Reduction Factor, D, changes.

\section{Impact of Linear Speed Dependence for Drag}

A formula for stopping distance was derived in the previous section under the assumption that drag forces exhibit a quadratic speed dependence. As noted earlier, this assumption was based on empirical results in runway friction tests that have shown a similar behavior for displacement drag due to water, slush, or snow accumulation. However, this assumption is not supported by the experimental results obtained during the current EMAS evaluation test. The observed speed dependence for drag in this test appeared to be closer to first-order than second-order. On the other hand, for practical reasons described earlier that had to do with resource constraints, the current data were acquired in a range of speeds that is considerably lower than the operational speed for which EMAS materials are ultimately to be evaluated. It is possible that the dependence of drag on speed is stronger in higher speed ranges. This remains an open research question, but because the current empirical evidence suggests a linear speed dependence for drag, in this section we re-derive the stopping-distance formula for the case of a linear dependence.

A surprising result is that the formula for stopping distance derived under the assumption of a linear speed dependence for drag is nearly identical to the formula that was derived earlier under the assumption of a quadratic speed dependence. The only difference is that the Drag Reduction Factor depends on the ratio of drag to friction in a slightly different way.

\section{A. Stopping Distance Derivation, Linear Speed Dependence for Drag}

The derivation of the formula for stopping distance when drag is proportional to speed follows the derivation presented earlier for an assumed quadratic speed dependence. As before, we begin with the total horizontal force, $F_{H}=m a$, where $m$ is the mass of the vehicle and $a$ is the horizontal acceleration. This force is just the sum of the force of friction, $m g \mu$, and a drag force that is proportional to velocity rather than velocity squared, with a proportionality constant we will call $K_{l}$ to denote its association with a first-order speed dependence:

$$
F_{H}=m a=-K_{1} V-m g \mu
$$

The vehicle will stop when the force in Eq. (84) has been applied through a distance sufficient to generate enough work to dissipate all of its kinetic energy, so as before the condition for stopping the aircraft in a distance, $R$, is

$$
1 / 2 m V^{2}=\int_{0}^{R} F_{H} d x
$$

Again we must express $F_{H}$ as a function of distance in order to perform the integral in Eq. (85). We begin by expressing Eq. (84) as the following differential equation:

$$
m \frac{d^{2} x}{d t^{2}}=-K_{1} \frac{d x}{d t}-m g \mu
$$

or:

$$
\frac{d^{2} x}{d t^{2}}+\left(\frac{K_{1}}{m}\right) \frac{d x}{d t}+g \mu=0
$$


Again we exploit the fact that acceleration is the derivative of speed with respect to time:

$$
V=\frac{d x}{d t} \rightarrow \frac{d^{2} x}{d t^{2}}=\frac{d V}{d t}
$$

so Eq. (87) becomes

$$
\frac{d V}{d t}+\left(\frac{K_{1}}{m}\right) V+g \mu=0
$$

Apply the chain rule to the derivative representing acceleration as in Eq. (18):

$$
\frac{d V}{d t}=\left(\frac{d V}{d x}\right)\left(\frac{d x}{d t}\right)=V\left(\frac{d V}{d x}\right)
$$

and insert this into Eq. (88):

$$
V\left(\frac{d V}{d x}\right)+\left(\frac{K_{1}}{m}\right) V+g \mu=0
$$

or

$$
V d V=-\left[\left(\frac{K_{1}}{m}\right) V+g \mu\right] d x
$$

Divide Eq. (90) by the bracketed term on the right:

$$
\frac{V d V}{\left[\left(\frac{K_{1}}{m}\right) V+g \mu\right]}=-d x
$$

We take the indefinite integral of both sides of Eq. (91).

$$
\int \frac{V d V}{\left[\left(\frac{K_{1}}{m}\right) V+g \mu\right]}=-\int d x
$$

The integral on the right is, of course, simply $-x$. The integral on the left is of a general form that can be evaluated as follows ${ }^{26}$ :

$$
\int\left(\frac{a+b x}{c+e x}\right) d x=\frac{b x}{e}+\frac{a e-b c}{e^{2}} \ln (c+e x)
$$


In this case, $x=V, a=0, b=1, c=g \mu$, and $e=K_{l} / m$, so Eq. (92) becomes

$$
\frac{m V}{K_{1}}-\left(\frac{m^{2} g \mu}{K_{1}^{2}}\right) \ln \left[g \mu+\left(\frac{K_{1}}{m}\right) V\right]=-x+\text { Const }
$$

where Const is a constant of integration. It is again convenient to express Const as $\ln (C)$, which we can do with no loss of generality, where $C$ is simply some constant yet to be evaluated.

We thus have

$$
\frac{m V}{K_{1}}-\left(\frac{m^{2} g \mu}{K_{1}^{2}}\right) \ln \left[g \mu+\left(\frac{K_{1}}{m}\right) V\right]=-x+\ln (C)
$$

Multiply through Eq. (95) by $K_{1}^{2} / m^{2}$ :

$$
\frac{K_{1} V}{m}-(g \mu) \ln \left[g \mu+\left(\frac{K_{1}}{m}\right) V\right]=-\left(\frac{K_{1}^{2}}{m^{2}}\right) x+\left(\frac{K_{1}^{2}}{m^{2}}\right) \ln (C)
$$

or

$$
\frac{K_{1} V}{m}-(g \mu) \ln \left(\frac{m g \mu+K_{1} V}{m}\right)=-\left(\frac{K_{1}^{2}}{m^{2}}\right) x+\left(\frac{K_{1}^{2}}{m^{2}}\right) \ln (C)
$$

Note that $\frac{K_{1} V}{m}=\ln \left(e^{\frac{K_{1} V}{m}}\right)$, so Eq. (97) can be rewritten as

$$
\ln \left(e^{\frac{K_{1} V}{m}}\right)-\ln \left(\frac{m g \mu+K_{1} V}{m}\right)^{g \mu}-\ln \left(C^{\frac{K_{1}^{2}}{m^{2}}}\right)=-\left(\frac{K_{1}^{2}}{m^{2}}\right) x
$$

or

$$
\ln \left[\frac{e^{\frac{K_{1} V}{m}}}{C^{\frac{K_{1}^{2}}{m^{2}}}\left(\frac{m g \mu+K_{1} V}{m}\right)^{g \mu}}\right]=-\left(\frac{K_{1}^{2}}{m^{2}}\right) x
$$

Exponentiation of both sides of Eq. (99) produces this:

$$
\frac{e^{\frac{K_{1} V}{m}}}{C^{\frac{K_{1}^{2}}{m^{2}}}\left(\frac{m g \mu+K_{1} V}{m}\right)^{g \mu}}=e^{-\left(\frac{K_{1}^{2}}{m^{2}}\right) x}
$$


We can evaluate the constant, $C$, from the boundary condition that requires $V$ in Eq. (100) to be $V_{0}$ when $x=0$ :

$$
\frac{e^{\frac{K_{1} V_{0}}{m}}}{C^{\frac{K_{1}^{2}}{m^{2}}}\left(\frac{m g \mu+K_{1} V_{0}}{m}\right)^{g \mu}}=1 \rightarrow C^{\frac{K_{1}^{2}}{m^{2}}}=\frac{e^{\frac{K_{1} V_{0}}{m}}}{\left(\frac{m g \mu+K_{1} V_{0}}{m}\right)^{g \mu}}
$$

Insert Eq. (101) into Eq. (100):

$$
\left(\frac{m g \mu+K_{1} V_{0}}{m g \mu+K_{1} V}\right)^{g \mu} e^{-\frac{K_{1}}{m}\left(V_{0}-V\right)}=e^{-\left(\frac{K_{1}^{2}}{m^{2}}\right) x}
$$

or

$$
\left(\frac{m g \mu+K_{1} V_{0}}{m g \mu+K_{1} V}\right)^{g \mu}=e^{-\left(\frac{K_{1}^{2}}{m^{2}}\right) x+\frac{K_{1}}{m}\left(V_{0}-V\right)}
$$

Take the natural logarithm of both sides of Eq. (103):

$$
(g \mu) \ln \left(\frac{m g \mu+K_{1} V_{0}}{m g \mu+K_{1} V}\right)=\frac{K_{1}}{m}\left(V_{0}-V\right)-\left(\frac{K_{1}^{2}}{m^{2}}\right) x
$$

When $x=R$, the stopping distance, $V=O$ and Eq. (104) becomes:

$$
(g \mu) \ln \left(\frac{m g \mu+K_{1} V_{0}}{m g \mu}\right)=\frac{K_{1} V_{0}}{m}-\left(\frac{K_{1}^{2}}{m^{2}}\right) R
$$

As when we assumed that drag varied as the square of speed, let $r$ represent the ratio of the drag force at EMAS entry speed to the force friction. That is, let $r=K_{1} V_{0} / m g \mu$. We then rewrite Eq. (105) as follows, after multiplying through by $m$ :

$$
(m g \mu) \ln (1+r)=K_{1} V_{0}-\left(\frac{K_{1}^{2}}{m}\right) R
$$

Divide through by $K_{l} V_{0}$, and again invoke the definition of $r$ :

$$
\frac{\ln (1+r)}{r}=1-\left(\frac{K_{1}}{m V_{0}}\right) R
$$

Solve Eq. (107) for $R$, the stopping distance:

$$
R=\frac{m V_{0}}{K_{1}}\left[1-\frac{\ln (1+r)}{r}\right]
$$


The term outside the brackets on the right of Eq. (108) can be written as follows:

$$
\frac{m V_{0}}{K_{1}}=\left(\frac{V_{0}^{2}}{2 g \mu}\right)\left(\frac{2 m g \mu}{K_{1} V_{0}}\right)=\frac{2 R_{f_{0}}}{r}
$$

where, as before, $R_{f_{0}}$ is the stopping distance for an initial speed of $V_{0}$, assuming only friction is in play (no drag). Inserting Eq. (109) into Eq. (108):

$$
R=\frac{2 R_{f_{0}}}{r}\left[1-\frac{\ln (1+r)}{r}\right]
$$

If drag depends on the square of speed as previously assumed, the stopping distance was seen to be the product of $R_{f_{0}}$ and a Drag Reduction Factor, $D$, which depended only on $r$, the ratio of drag force to friction force. Equation (110) reveals that the same is true when the drag features only a first-order speed dependence. That is, the stopping distance is $R_{f_{0}} D$ in either case, where $D$, the Drag Reduction Factor, is as follows:

$$
D= \begin{cases}\frac{\ln (1+r)}{r} & \text { Drag } \propto V^{2} \\ \frac{2}{r}\left[1-\frac{\ln (1+r)}{r}\right] & \text { Drag } \propto V\end{cases}
$$

We showed in the analysis that was based on a quadratic speed dependence for drag that the Drag Reduction Factor is a dimensionless number bounded by 0 and 1 , which can be interpreted as the degree of further reduction in stopping distance from the friction-only case that is achieved by introducing drag. We now demonstrate that the Drag Reduction Factor is also bounded by 0 and 1 when drag is directly proportional to speed. It is necessary to show this for any $r$, since adding drag must result in a shorter stopping distance than the friction-only case for any ratio of drag to friction, no matter how drag depends on speed.

We evaluate $D$ for the case of linear $V$ dependence in the limit as $r$ approaches zero and again in the limit as $r$ approaches infinity. As was the case for a quadratic speed dependence for drag, $D$ approaches an indeterminate form in both cases, but we can circumvent this by again invoking L'Hopital's Rule, which states that as $x$ approaches some limit, $c$, the ratio of $f(x) / g(x)$ approaches the ratio of the derivatives of $f(x)$ and $g(x)$ if that ratio exists. Begin by expanding the version of $D$ for linear speed dependence from Eq. (111):

$$
D=\frac{2}{r}\left[1-\frac{\ln (1+r)}{r}\right]=\frac{2 r-2 \ln (1+r)}{r^{2}}
$$

By L'Hopital's Rule, we divide the derivative of the numerator of Eq. (112) by the derivative of the denominator:

$$
\lim _{r \rightarrow c} D=\frac{2-2\left(\frac{1}{1+r}\right)}{2 r}=\left.\frac{1}{1+r}\right|_{r=c}=\frac{1}{1+c}
$$


just as in Eq. (54). And therefore just as before, we have

$$
\lim _{r \rightarrow 0} D=1
$$

and

$$
\lim _{r \rightarrow \infty} D=0
$$

so $0 \leq D \leq 1$ for all values of $r$, as required.

We see that the formula for stopping distance has the same general form whether drag depends on speed or the square of speed. It can be expressed in either case as the product of a well-known formula for stopping distance when only friction is in play, and a Drag Reduction Factor, D. In either case, The Drag Reduction Factor is a function of $r$, the ratio of drag to the force of friction, but the form of the relationship between $D$ and $r$ is slightly different for the linear and quadratic speed dependence cases as Eq. (111) indicates. This difference results in a somewhat different expression for the variance in stopping distance as a function experimental force measurement errors, as will now be demonstrated.

\section{B. Uncertainty in Stopping Distance, Linear Speed Dependence for Drag}

Assume as before that we are only interested in the uncertainty in stopping distance under operational conditions in which drag dominates and frictional forces make only a negligible contribution to the total horizontal force. In that case, $F_{H}>>f$; i.e., $r>>1$, and:

$$
r \approx 1+r \approx \frac{F_{H}}{f}
$$

Then

$$
D=\frac{2}{r}\left[1-\frac{\ln (r)}{r}\right]=\frac{2 f}{F_{H}}\left[1-\frac{f}{F_{H}} \ln \left(\frac{F_{H}}{f}\right)\right]
$$

and

$$
R=\frac{V_{0}^{2} F_{V}}{g F_{H}}\left[1-\frac{f}{F_{H}} \ln \left(\frac{F_{H}}{f}\right)\right]=2 \lambda\left(\frac{F_{V}}{F_{H}}\right)\left[1-\frac{f}{F_{H}} \ln \left(\frac{F_{H}}{f}\right)\right]
$$

where we as before we use the symbol $\lambda=\frac{V_{0}^{2}}{2 g}$ to simplify the notation.

We will again invoke the error propagation formula first given in Eq. (3), which depends on the derivatives of the stopping distance formula with respect to $F_{V}, F_{H}$, and $f$. To facilitate the differentiation, we rewrite the stopping distance formula as follows:

$$
R=2 \lambda\left(\frac{F_{V}}{F_{H}}\right)\left[1-\frac{f}{F_{H}} \ln \left(\frac{F_{H}}{f}\right)\right]=2 \lambda F_{V} F_{H}^{-1}\left\{1-f F_{H}^{-1}\left[\ln \left(F_{H}\right)-\ln (f)\right]\right\}
$$

If we again introduce the simplifying nomenclature:

$$
m=\ln \left(\frac{F_{H}}{f}\right)
$$


the required derivatives of stopping distance can be summarized as follows:

$$
\begin{gathered}
\frac{\partial R}{\partial F_{V}}=\left(\frac{2 \lambda F_{V}}{F_{H}^{2}}\right)\left\{\left(\frac{F_{H}}{F_{V}}\right)\left[1-\left(\frac{f}{F_{H}}\right) m\right]\right\} \\
\frac{\partial R}{\partial F_{H}}=\frac{2 \lambda F_{V}}{F_{H}^{2}}\left[-1+\frac{f}{F_{H}}\left(m^{2}-1\right)\right] \\
\frac{\partial R}{\partial f}=-\left(\frac{2 \lambda F_{V}}{F_{H}^{2}}\right) m
\end{gathered}
$$

We again introduce the nomenclature that distinguishes large-force experimental errors from the errors in estimating the much smaller force of friction, and again assume that the uncertainty in horizontal and vertical largeforce measurements is the same:

$$
\sigma_{F}^{2}=\sigma_{F_{V}}^{2}=\sigma_{F_{H}}^{2}
$$

Inserting Eqs. (117) into Eq. (62) with the indicated notational changes produces this result:

$$
\sigma_{R}^{2}=\left(\frac{2 \lambda F_{V}}{F_{H}^{2}}\right)^{2}\left\langle\left\{\left(\frac{F_{H}-m f}{F_{V}}\right)^{2}+\left[\left(m^{2}-1\right)\left(\frac{f}{F_{H}}\right)\right]^{2}\right\} \sigma_{F}^{2}+m \sigma_{f}^{2}\right\rangle
$$

For the high-speed conditions that are of most interest, the total horizontal force will be large compared to the force of friction and Eq. (118) can be simplified to good approximation as follows:

$$
\sigma_{R}^{2}=\left(\frac{2 \lambda F_{V}}{F_{H}^{2}}\right)^{2}\left[\left(\frac{F_{H}}{F_{V}}\right)^{2} \sigma_{F}^{2}+m \sigma_{f}^{2}\right]
$$

or

$$
\sigma_{R}^{2}=\left(\frac{2 \lambda}{F_{H}}\right)^{2}\left[\sigma_{F}^{2}+m\left(\frac{F_{V}}{F_{H}}\right)^{2} \sigma_{f}^{2}\right]
$$

The standard error for stopping distance is just the square root of the variance given in Eq. (120):

$$
\sigma_{R}=\frac{2 \lambda}{F_{H}} \sqrt{\sigma_{F}^{2}+m\left(\frac{F_{V}}{F_{H}}\right)^{2} \sigma_{f}^{2}}
$$

A comparison of this result with Eq. (72) reveals that if drag is a linear function of speed, the standard error in the estimate of stopping distance is slightly more complex than if the speed dependence is quadratic. In both cases the standard error depends on the variance in experimental force measurements, but in the case of a linear speed dependence for drag we cannot justify ignoring the large-force errors as we were able to do for the case in which drag depends on the square of speed. We discuss the reason for this, and other points, in the next section. 


\section{Discussion}

A number of unanticipated results occurred as this study unfolded along the following lines: A relationship between stopping distance and the forces measured in this EMAS evaluation experiment was first derived for the purpose of propagating experimental force measurement errors into an estimate of the standard error in stopping distance. The intent was to reveal how the uncertainty in stopping distance depends on these measurement errors, and to highlight any conditions for which special care might be required in the force measurements to ensure high quality estimates of stopping distance in future experiments.

After the test and prior to the analysis there was an assumption that stopping distance was a function of the sum of large drag forces plus a much smaller and essentially negligible force of rolling friction, especially for the high EMAS entry speeds of particular concern. This assumption was predicated in part on a supposition that displacement drag would display a relatively strong (quadratic) dependence on speed, while the force of friction was known to be a relatively small fraction of applied load that was independent of speed.

The derivation of the stopping distance formula revealed an unanticipated relationship between stopping distance and the ratio of drag force to the force of friction, rather than their sum, which has two important implications. The first is that the force of friction cannot simply be dismissed as a negligible augmentation of a dominant drag force. Stopping distance is influenced as much by the force of friction as by the force of drag, notwithstanding the substantially larger magnitude of drag. This then implied that the force of friction and the force of drag must each be estimated separately. Stopping distance is apparently not a simple function of some total horizontal retarding force. Furthermore, the error propagation analysis that originally motivated the stopping distance derivation revealed that uncertainty in stopping distance was a stronger function of experimental errors in the measurement of friction than of drag. This unanticipated result was true notwithstanding the degree to which drag dominates the total horizontal force, and in fact the smaller friction is, the greater the role of friction measurement errors in estimating the uncertainty in stopping distance.

These insights lead to an attempt to segregate drag and friction forces in the current experiment, by the following technique: Horizontal forces on the test wheel measured as it traversed an EMAS test bed at very shallow immersion depths were attributed to friction only, since drag depends on the frontal area of EMAS material presented to the wheel This friction-only assumption was tested by comparing the force measurements acquired under such shallowimmersion conditions at two different speeds. Since drag depends on speed and friction does not, the fact that no difference could be resolved between the lower- and higher-speed horizontal force measurements for these very shallow immersions was interpreted as confirmation that drag was negligible under those conditions, and the measured horizontal forces could be attributed entirely to friction.

These pure friction forces were used to estimate the coefficient of friction for the two candidate EMAS materials under evaluation. The friction coefficient is simply the ratio of the force of friction to the vertical load force. It can be used to estimate friction forces in other circumstances, by simply multiplying it by the vertical load in those circumstances.

A series of higher-immersion runs was executed in which both drag and friction were in play. The force of friction was estimated for those runs from measured vertical load force data and the coefficient of friction determined previously from the friction-only runs. These friction force estimates were then subtracted from measured values of the total horizontal force in order to isolate the drag force.

This isolation of drag forces resulted in another unanticipated result. The dependence of drag on speed was examined by comparing the ratio of drag values acquired at higher and lower speeds with the ratio of those speeds. Because of the assumed quadratic speed dependence, it was anticipated that the ratio of higher-speed and lowerspeed drag measurements would be the same as the square of the speed ratio. However, the drag ratio was observed to be much closer to the ratio of speeds than to the ratio of the square of the speeds. This suggested a linear speed dependence for drag rather than the quadratic speed dependence upon which the derivation of the stopping distance formula had been based.

It was the formula for stopping distance based on a quadratic speed dependence for drag that had predicted the unanticipated dependence of stopping distance on the ratio of drag to friction rather than the sum, and which had motivated the segregation of drag and friction forces in the first place. Since this relationship was now in doubt, the formula for stopping distance as a function of measured forces was re-derived, this time for a linear dependence of drag on speed. Again surprisingly, the same dependence of stopping distance on the ratio of drag to friction was observed, with only a slightly different functional form. That is, in either case the stopping distance could be expressed as the product of a simple and well-known formula for stopping distance when only friction is in play, and a dimensionless Drag Reduction Factor, D, in the range of 0 to 1 that quantifies the further reduction in stopping distance achieved when drag is introduced. Whether the dependence of drag on speed is linear or quadratic, D is a 
function only of the ratio of the drag force at EMAS entry to the force of friction. The only difference is that D is a slightly different function of this ratio for the two speed dependencies, as Eq. (111) indicates.

Figure 4 illustrates how the Drag Reduction Factor depends on the behavior of drag as a function of speed. For a given ratio of drag force at EMAS entry speed to force of friction, a quadratic speed dependence for drag would result in a slightly greater reduction in stopping distance than if the speed dependence was linear.

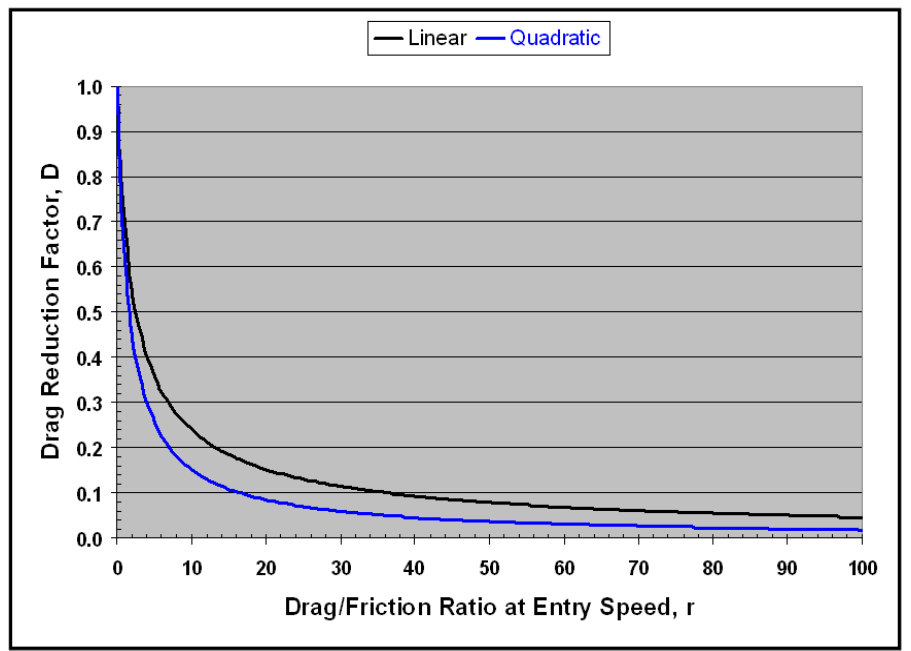

Figure 4. Drag Reduction Factors when Displacement Drag is a Linear or Quadratic Function of Speed.

Figure 4 reveals some important insights about the impact on stopping distance of adding drag to the ubiquitous force of friction. The first is that drag has a greater effect earlier and a lesser effect later. That is, a small increase in drag from the no-drag, friction-only case has a dramatic impact in reducing stopping distance, but the more drag that is in play, the less effective additional drag becomes. Once the drag force at EMAS entry speed is large enough relative to the force of friction that this ratio, $r$, is well to the right of the knee in Fig. 4, adding drag becomes a relatively ineffective tactic for achieving substantial further reductions in stopping distance.

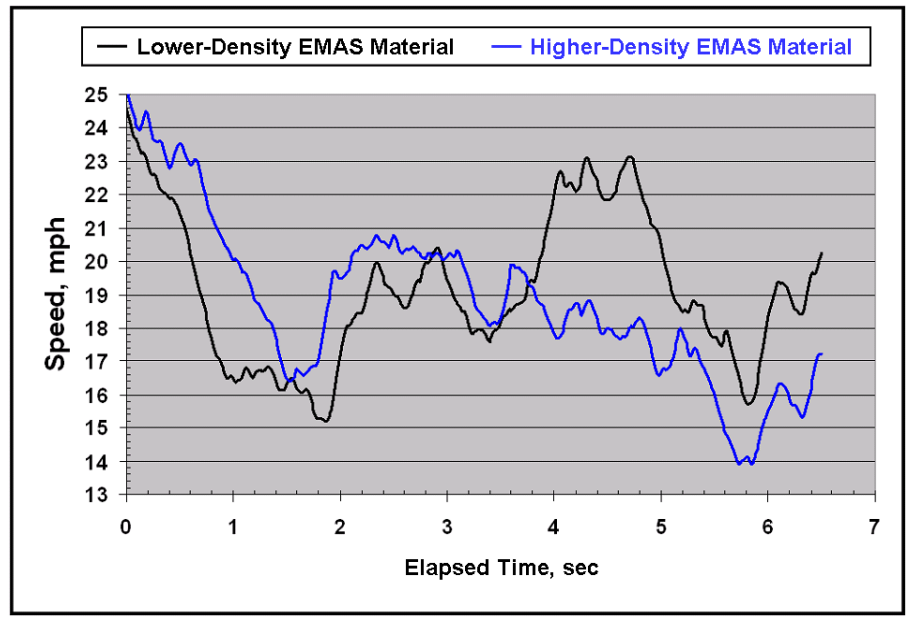

Figure 5: Impact of EMAS material on Speed of MTTF

The effectiveness of drag as an agent for dissipating kinetic energy can be seen in Fig. 5, which displays the speed time histories for MTTF runs through the lower-density and higher-density EMAS material with a test-tire immersion depth of less than four inches. The vehicle tires of the MTTF straddled a narrow test trench filled with EMAS material in each case, so that only the trailing test wheel (Figs. 1 and 6) encountered the EMAS material. 
Notwithstanding the relatively shallow immersion of the test wheel, drag induced by the EMAS material was able to reduce the speed of the 18,000-pound MTTF from about $25 \mathrm{mph}$ to about $16 \mathrm{mph}$ in less than two seconds. This corresponds to a dissipation of more than half of the kinetic energy of the MTTF, under conditions in which the operator was attempting to maintain a constant speed by applying pressure to the accelerator peddle. The energy reduction is likely to have been even greater had there been no accelerating forces on the MTTF, and greater still without the restrictions on test-wheel immersion depth that are discussed below.

The MTTF operator experienced no trouble maintaining a constant speed over asphalt, where only friction and no drag was in play. Figures 4 and 5 are consistent, and suggest that adding even a little drag can result in a substantial reduction in kinetic energy.

Figure 4 also illustrates something interesting about the impact of force measurement errors on the uncertainty in estimates of stopping distance. Under the most interesting practical conditions, which involve EMAS entry speeds large enough for the quantity $r$ in Fig. 4 to be well to the right of the knee, the change in stopping distance is a relatively weak function of the change in EMAS entry drag. That is, the magnitude of the slope of $D$ is relatively small. In this region, rather large errors in the estimates of drag forces will have relatively minor effects on the estimates of stopping distance. This suggests that for practical purposes, a precise estimate of drag at a given EMAS entry speed is not a prerequisite for a good estimate of stopping distance. On the other hand, to estimate stopping distance the Drag Reduction Factor plotted in Fig. 4 is multiplied by a formula for friction-only stopping distance that is inversely proportional to friction coefficient, which is directly proportional to measured friction force values. This suggests that for the relatively low levels of friction coefficient anticipated under the no-braking conditions considered in this analysis, the uncertainty in stopping distance will be a relatively sensitive function of experimental errors in friction force measurements. This is supported by Eqs. (69) through (72) and the ensuing discussion.

Although the general features of how stopping distance depends on the forces of friction and drag are the same for either a linear or quadratic speed dependence for drag as Fig. 4 indicates, the true dependence of drag on speed under operational conditions of special interest remains an open research question. There are two reasons for this. The first is that the $5 \mathrm{mph}$ to $30 \mathrm{mph}$ speed range specified by the PANYNJ for this preliminary test is well below the 70-knot (80.6 mph) upper speed limit for which the FAA requires effective EMAS performance. It is therefore possible that something akin to Stokes Drag is in play at lower speeds, with its linear dependence on speed as observed in this experiment, while at higher speeds there is a transition to something that behaves more like Rayleigh Drag, with its quadratic speed dependence.

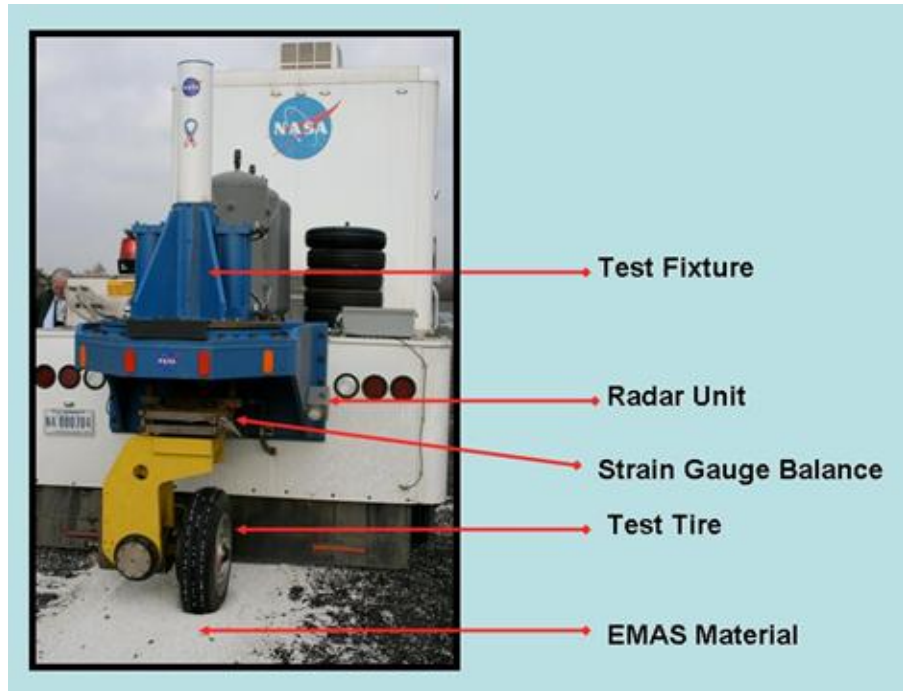

Figure 6. MTTF Test Wheel Assembly

The second reason that drag may depend on speed in a different way under operational conditions of interest than in this test is that full immersion depths were not realized, and the speed dependence for drag may be a function of immersion depth. Test wheel immersion depths were limited in this test to a few inches by a mechanical stop built into the MTTF test wheel assembly. The purpose of the mechanical stop was to keep the wheel mount hardware (yellow component in MTTF test wheel assembly of Fig. 6 up and out of the EMAS material, so that only forces on 
the test wheel would be measured by the strain gauge balance shown in that figure. With an MTTF design modification that would facilitate full-immersion runs, it is possible that a quadratic dependence of drag on speed might be observed.

Principal lessons learned in the present study include the fact that the dependence of drag on speed is an important factor in estimating stopping distance, and since this dependence may vary with such factors as immersion depth and mean speed, it needs to be evaluated under the full-speed, full-immersion conditions that are of most practical interest. A second important lesson learned is that since stopping distance depends on the ratio of displacement drag at EMAS entry speed to the force of friction, and not the sum of those two forces, it is just as important to measure friction under conditions of negligible drag as it is to measure drag under conditions of negligible friction. This would involve very shallow immersion depths and very low speeds, since drag is a function of the frontal area of material presented to the wheel and is also a function of speed.

\section{Summary of Results}

This study provided a number of unanticipated insights into the behavior of an aircraft during a runway overrun incident involving an Engineered Materials Arresting System. These insights are related to the relative importance of friction and drag in such an incident, the dependence of drag on speed, the precision requirements in force measurements made as part of an EMAS materials evaluation experiment, and the unexpectedly simple relationship between stopping distance and the forces that are responsible for the work that dissipates the aircraft's kinetic energy when both friction and drag are in play.

An analytical investigation of stopping distance as a function of entry speed and forces on the test wheel lead to two different formulations for a stopping distance formula. One was based on the assumption of a quadratic dependence of drag on speed that is similar to Rayleigh Drag, and one was based on the assumption of a linear dependence of drag on speed that is similar to Stokes Drag. A linear speed dependence for drag was observed for the low-speed, shallow-immersion runs conducted in this test. However, it was not possible to achieve full test wheel immersion depths because of a safety constraint designed to keep part of the test wheel assembly elevated, and realestate constraints (limited space for acceleration/deceleration) also prevented an evaluation of the EMAS materials at the full 70-knot speed specified by the FAA. The true speed dependence for drag under operational conditions therefore remains an open research question, and may in fact be quadratic notwithstanding the linear dependence observed under the limited conditions of this test. However, the dependence of stopping distance on measured forces was found to be similar for either a linear or quadratic dependence of drag on speed, and the essential findings of this test are the same in either case.

Principal findings of this study are summarized as follows:

- Stopping distance is not a simple function of the total horizontal force experienced by a wheel as it enters an EMAS runway extension. That is, the forces of friction and displacement drag do not simply add to produce a retarding force that can be used to forecast stopping distance.

- When drag and friction are both in play, stopping distance for a given entry speed into an EMAS is the same as it would be if friction was in play and drag was not, except that this friction-only distance is further reduced by a dimensionless constant between 0 and 1 that quantifies the role of drag. This constant was named the Drag Reduction Factor, $D$, in this report.

- The Drag Reduction Factor is a function of the ratio of the two horizontal force components. It depends on the ratio of the drag force at $V_{0}$, the EMAS entry speed, to the speed-independent force of friction.

- The form of the Drag Reduction Factor depends on whether displacement drag is proportional to speed or the square of speed. An anticipated quadratic speed dependence was not confirmed by the data, which suggested a linear speed dependence instead. However, because of certain resource and safety constraints described in the paper, the data were acquired at lower speeds and more shallow immersion depths than will apply under typical operational conditions, for which the speed dependence may be stronger.

- Drag generates a substantial further reduction in stopping distance beyond that which is attributable to rolling friction only. Further reductions of a factor of 5 to 10 or more beyond those achievable with rolling friction alone appear to be practical with realistically anticipated levels of drag. 
- Drag effects accrue early. With even a modest addition of drag, the stopping distance drops substantially below what it would have been if only rolling friction were in play.

- The impact that additional drag has on stopping distance is a monotonically decreasing function of the amount of drag already in play. Adding more drag therefore has a greater effect when relatively little drag is in play and rather less of an effect once there is already a substantial amount of drag.

- The standard error in estimating stopping distance is a weighted sum of the standard experimental errors in measuring friction and the larger forces of horizontal drag and vertical load. Uncertainty in stopping distance is a stronger function of experimental errors in estimating friction than in estimating either horizontal drag or vertical load, notwithstanding how much greater the drag and load forces tend to be than the force of friction under typical operating conditions.

\section{Acknowledgments}

The data upon which the analysis in this report rests were acquired by the Structural Dynamics Branch of NASA Langley Research Center, in collaboration with the Port Authority of New York and New Jersey. Funding was provided by the Port Authority and by NASA. Technical support was provided by the Research Services Directorate and the Aerospace Testing Techniques Branch of Langley Research Center. The contributions of Marco Pirozzi, John Costulis, Lucille Crittenden, Scott Runnels, Jose Vega, and Kathy Barnstorff are gratefully acknowledged.

\section{References}

${ }^{1}$ Thompson, W. C., "Investigation of Water-Pond Arresting of a Dynamic Model of a Jet Transport", NASA TN-D 732, December 1960.

${ }^{2}$ Bade, E., "Soft-Ground Arresting of Civil Aircraft", Royal Aircraft Establishment Technical Report 68032, February 1968.

${ }^{3}$ Randall, T.G., "Preliminary Feasibility Study of the Arresting of Aircraft in a Foamed Plastic Overrun Area", Royal Aircraft Establishment Technical Memorandum Naval 213. 1970.

${ }^{4}$ Gwynne, G.M., “Aircraft Arresting Using Foamed Plastic Overrun Areas”, Royal Aircraft Establishment NAD Note No. 282, March 1971.

${ }^{5}$ Bade, E. and Minter, E.M., "Soft-Ground Arresting of Civil Aircraft - Scaled Model VC-10 Tests in Gravel and Sintered Fuel Ash Pellets”, Royal Aircraft Establishment Technical Report 71015, 1971.

${ }^{6}$ Gwynne, G.M., "Use of Foamed Plastics as Emergency Aircraft Arresters - Study of the Arresting Effects for Different Groups of Aircraft on Selected Runways", Royal Aircraft Establishment Technical Memorandum Naval $217,1972$.

${ }^{7}$ Gwynne, G.M., "Urea Formaldehyde Foamed Plastic Emergency Arresters for Civil Aircraft", Royal Aircraft Establishment Technical Report 74002, February 1974.

${ }^{8}$ Phillips, N.S. and Cook, R.F, "Aircraft Operation on Soil Surfaces - Computer Routine Revisions and Improvement", Volume I, ESL-TR-82-29, March 1984.

${ }^{9}$ Cook, R.F., “Aircraft Operating on Soil-Prediction Techniques”, Grouping 1: Volume 1, Discussion, ESL-TR-84-04, July 1985.

${ }^{10}$ Cook, R.F., "Soft-Ground Aircraft Arresting Systems", Final Report for Period September 1986 to August 1987, DOT/FAA/PM-87/27, August 1987. 1993.

${ }^{11}$ Davis, P.A. and Stubbs, S.M., "Studies of Shuttle Orbiter Arrestment System”, NASA Technical Paper 3370, December

${ }^{12}$ Federal Aviation Administration Advisory Circular No. 150/5220-22A, September 2005.

${ }^{13}$ Space Act Agreement SAA1-894, "Agreement Between the National Aeronautics and Space Administration and the Port Authority of New York and New Jersey”, October 2007.

${ }^{14}$ DeLoach, R., "Improved Quality in Aerospace Testing Through the Modern Design of Experiments (invited)". AIAA 20000825. 38th AIAA Aerospace Sciences Meeting and Exhibit. Reno, NV. Jan 2000.

${ }^{15}$ DeLoach, R., "Applications of the Modern Design of Experiments at NASA Langley Research Center (Invited)" Proceedings of the American Statistical Association, Section on Physical and Engineering Sciences [CD-ROM], New York, NY: American Statistical Association, 2002.

${ }^{16}$ DeLoach, R., "Formal Experiment Design as a Tool to Automate Aerospace Ground Testing (Invited)". Tenth Annual Spring Research Conference on Statistics in Industry and Technology. June 4-6, 2003 University of Dayton.

${ }^{17}$ DeLoach, R., "The Modern Design of Experiments: An Emerging Knowledge Management Approach to Aerospace Testing”. AIAA-2005-7076. AIAA Engineering InfoTech@ Aerospace Conference. Arlington, VA 26 - 29 September, 2005

${ }^{18}$ DeLoach, R., "Propagation of Computational Uncertainty Using the Modern Design of Experiments (Invited)", Symposium on Computational Uncertainty, organized by the Applied Vehicle Technology Panel (AVT-147) of the North Atlantic Treaty Organization Research and Technology Agency. Athens, Greece, 3 to 6 December 2007.

${ }^{19}$ DeLoach, R., "Bayesian Inference in the Modern Design of Experiments", AIAA-2008-0847. 46th AIAA Aerospace Sciences Meeting and Exhibit, Reno, NV Jan 7-10, 2008. 
${ }^{20}$ DeLoach, R., Marlowe, J. M., Crittenden, L. H., Vega, J. A., Runnells, S. R., and Yager, T. J., “An Evaluation of Materials for Engineered Materials Arresting Systems Using the Modern Design Of Experiments". Report to the Port Authority of New York and New Jersey, September 2008.

${ }^{21}$ Coleman, H. W. and Steele, W. G. (1989). Experimentation and Uncertainty Analysis for Engineers. New York: Wiley.

${ }^{22}$ Bevington, P.R. and Robinson, D.K. $\left(1992,2^{\text {nd }}\right.$ Ed). Data Reduction and Error Analysis for the Physical Sciences. New York: McGraw-Hill.

${ }^{23}$ Horne, Walter B., and Dreher, Robert C., Phenomena of Pneumatic Tire Hydroplaning. NASA TN D-2056, 1963.

${ }^{24}$ Sugg, Robert W., The Drag of a Large and Small Pneumatic Tyre Travelling Through Water, Slush and Snow. S \& T Memo 9/66, Oct. 1966.

${ }^{25}$ Horne, Walter B., Joyner, Upshur T., and Leland, Trafford J.W., Studies of the Retardation Force Developed on an Aircraft Tire Rolling in Slush or Water. NASA TN D-552, 1960.

${ }^{26}$ Gradshteyn, I.S. and Ryzhik, I.M. $\left(1965,4^{\text {th }}\right.$ Ed prepared by Geronimus, YU. V. and Tseytlin, M. Yu., translated from the Russian by Scripta Technica, Inc, translation edited by Alan Jeffrey), Table of Integrals, Series, and Products. New York: Academic Press. 